\title{
CD1b-autoreactive T cells contribute to hyperlipidemia-induced skin inflammation in mice
}

\author{
Sreya Bagchi, ${ }^{1}$ Ying He, ${ }^{1}$ Hong Zhang, ${ }^{1}$ Liang Cao, ${ }^{1}$ Ildiko Van Rhijn, ${ }^{2,3}$ D. Branch Moody, ${ }^{2}$ Johann E. Gudjonsson, ${ }^{4}$ and Chyung-Ru Wang ${ }^{1}$ \\ 'Department of Microbiology and Immunology, Feinberg School of Medicine, Northwestern University, Chicago, Illinois, USA. ²Department of Medicine, Division of Rheumatology, Immunology and Allergy, \\ Brigham and Women's Hospital, Harvard Medical School, Boston, Massachusetts, USA. ${ }^{3}$ Department of Infectious Diseases and Immunology, School of Veterinary Medicine, Utrecht University, Utrecht, \\ Netherlands. ${ }^{4}$ Department of Dermatology, University of Michigan, Ann Arbor, Michigan, USA.
}

\begin{abstract}
A large proportion of human T cells are autoreactive to group 1 CD1 proteins, which include CD1a, CD1b, and CD1c. However, the physiological role of the CD1 proteins remains poorly defined. Here, we have generated a double-transgenic mouse model that expresses human CD1b and CD1c molecules (hCD1Tg) as well as a CD1b-autoreactive TCR (HJ1Tg) in the ApoE-deficient background (hCD1Tg HJ1Tg Apoe ${ }^{-/-}$mice) to determine the role of CD1-autoreactive T cells in hyperlipidemia-associated inflammatory diseases. We found that hCD1Tg HJ1Tg $A p o e^{-/-}$mice spontaneously developed psoriasiform skin inflammation characterized by $T$ cell and neutrophil infiltration and a Th17-biased cytokine response. Anti-IL-17A treatment ameliorated skin inflammation in vivo. Additionally, phospholipids and cholesterol preferentially accumulated in diseased skin and these autoantigens directly activated CD1b-autoreactive HJ1 T cells. Furthermore, hyperlipidemic serum enhanced IL-6 secretion by CD1b+ DCs and increased IL-17A production by HJ1 T cells. In psoriatic patients, the frequency of CD1b-autoreactive T cells was increased compared with that in healthy controls. Thus, this study has demonstrated the pathogenic role of CD1b-autoreactive T cells under hyperlipidemic conditions in a mouse model of spontaneous skin inflammation. As a large proportion of psoriatic patients are dyslipidemic, this finding is of clinical significance and indicates that self-lipid-reactive T cells might serve as a possible link between hyperlipidemia and psoriasis.
\end{abstract}

\section{Introduction}

Hyperlipidemia is a condition characterized by elevated plasma lipid levels. LDL and triglycerides contribute to human disease in many organs. Approximately one-third of Americans show signs of hyperlipidemia or obesity (1). Excess plasma lipids have been associated with several chronic inflammatory conditions, such as cardiovascular diseases, including atherosclerosis (2). Interestingly, elevated serum lipid levels have also been reported in psoriasis patients, who have an increased risk of developing obesity and cardiovascular ailments (3-6). Even though the mechanisms that underlie the links among psoriasis, hyperlipidemia, and atherosclerosis remain unclear, systemic inflammation is a hallmark of patients with all these conditions (5). Psoriasis, a primarily $\mathrm{T}$ cell-driven autoimmune disease, affects approximately $1 \%$ to $3 \%$ of humans $(3,7)$. Activation and subsequent cytokine secretion by self-peptide-reactive T cells contribute to disease maintenance (8, 9). Further, T cell responses in psoriasis patients are largely Th17 and Th1 biased $(8,9)$. In fact, anti-TNF is a mainstay of therapy for psoriasis, and anti-IL-17A therapy was recently approved as a first-line systemic treatment $(10,11)$. While the involvement of peptide-reactive $\mathrm{T}$ cells has been well defined (12-15), the role of lipid-reactive $\mathrm{T}$ cells in psoriasis remains largely unknown. Since psoriasis and cardiovascular disease are correlated with

Conflict of interest: The authors have declared that no conflict of interest exists

Submitted: December 9, 2016; Accepted: March 3, 2017.

Reference information: / Clin Invest. 2017;127(6):2339-2352.

https://doi.org/10.1172/JCI92217. hyperlipidemia and recent studies have identified CD1-restricted self-lipid-reactive $\mathrm{T}$ cells in the blood or skin of humans (16-19), we developed a new mouse model for the study of human CD1restricted $\mathrm{T}$ cell responses to self-lipids in vivo.

CD1 molecules are divided into group 1 (CD1a, CD1b, and CD1c) and group 2 (CD1d) based on sequence homology (20). The role of CD1d-restricted NKT cells under homeostatic and inflammatory conditions has been extensively studied in mice (21). Since mice express only CD1d, very little is known about the in vivo function of group 1 CD1-restricted T cells (22). All 4 types of CD1 molecules are expressed on double-positive $\left(\mathrm{CD} 4^{+} \mathrm{CD} 8^{+}\right)$ thymocytes $(23,24)$. While CD1d is expressed on both hematopoietic and nonhematopoietic cells in the periphery, group 1 CD1 expression is restricted to antigen-presenting cells $(23,24)$. Over the years, it has become apparent that autoreactivity is a common feature of CD1-restricted T cells (16, 25-29). Further, T cells that are measurably autoreactive to group $1 \mathrm{CD} 1$ proteins are abundant in human blood $(16,18)$. CD1a autoreactive T cells have been shown to recognize apolar "headless" skin-derived lipid antigens (17). A recent study also demonstrated that CD1a-restricted T cells responded to neolipid antigens generated by phospholipase $\mathrm{A}_{2}$ activity in psoriasis patients (30). CD1b-autoreactive T cells can recognize phospholipids $(17,19)$, and a higher frequency of sulfatide and monosialotetrahexosylganglioside-specific (GM1-specific) $\mathrm{T}$ cells was detected in multiple sclerosis patients compared with normal controls (26). Moreover, CD1c-restricted T cells specific for cholesteryl esters have recently been identified (31). Finally, most CD1d-restricted NKT cells also exhibit autoreactivity and 
A

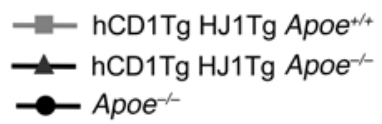

Percent weight change

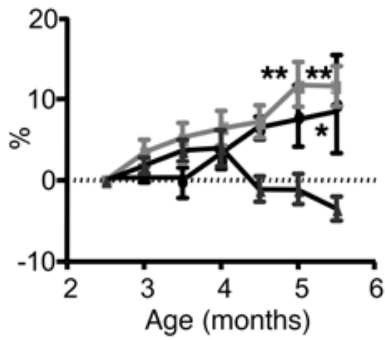

B

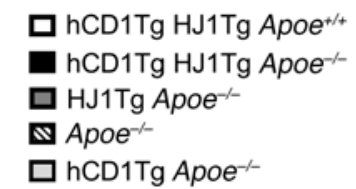

Dermatitis incidence
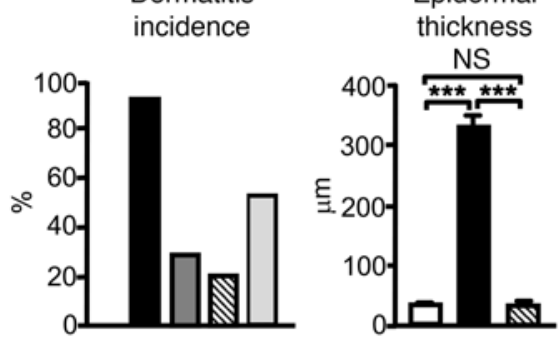
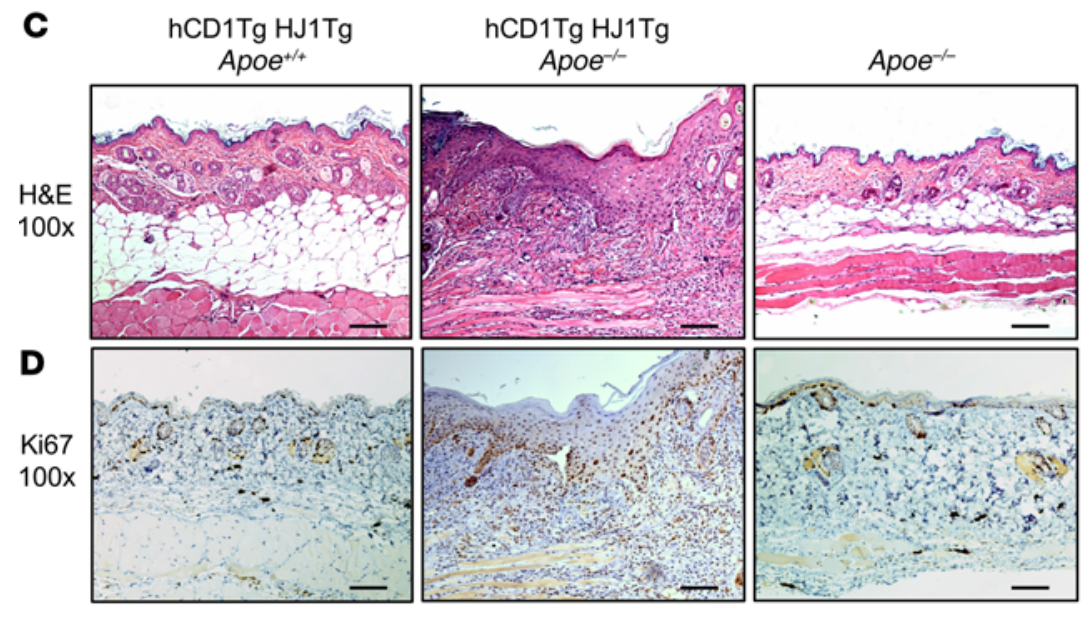

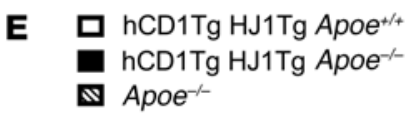

Skin S100 mRNA level

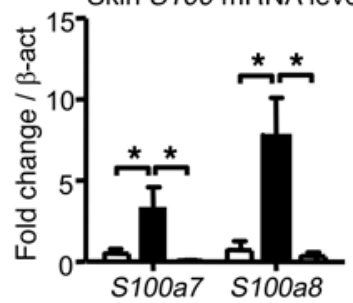

F

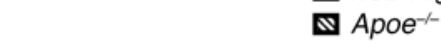

Aortic root plaque area

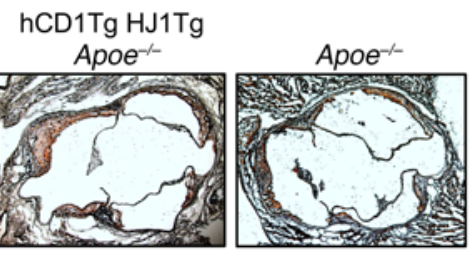

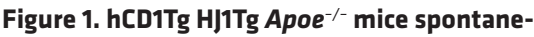
ously develop skin inflammation. (A) Mice were weighed beginning at 2.5 months of age up to 5.5 months of age. Percentage change in body weight was calculated over the time period $(n=4-10)$. (B) Mice were monitored from 20 to 25 weeks for the development of dermatitis and percentages of incidence recorded (left); epidermal thickness was also quantified (right; $n=11-14$ ). (C and D) Representative H\&E (C) and Ki67 (D) staining of skin sections from 6-month-old mice. Scale bars: $100 \mu \mathrm{m}$. (E) mRNA analysis of S100a proteins in the skin of indicated mice $(n=3-5)$. (F) Representative oil red 0 -stained sections from aortic root of

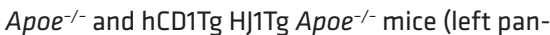
el) are shown, and bar graph depicts the mean + SEM of aortic root plaque area from indicated mice $(n=8-9) .{ }^{* *} P<0.005 ;{ }^{* *} P<0.01 ;{ }^{*} P<$

0.05 . Statistical analyses were performed using 1-way ANOVA followed by Bonferroni's post-hoc test for 3-group comparisons and Student's $t$ test for 2-group comparisons. secrete both Th1- and Th2-type cytokines following activation (29). Together, these studies have provided rapid progress in identifying lipid autoantigens as well as proof of principle that human $\mathrm{T}$ cells recognize self-lipids in vivo, raising the possibility that they play pathogenic roles in autoimmune diseases.

NKT cells contribute to psoriasis in both mice and humans (32) via IFN- $\gamma$ secretion upon interaction with CD1d-expressing keratinocytes $(33,34)$. However, group $1 \mathrm{CD} 1$ proteins have not been investigated in this regard owing to lack of tractable animal models and lack of information about CD1 expression in human disease. Interestingly, a recent study showed that group 1 CD1 mRNA is increased in human psoriatic lesions (35) and that
CD1a blockade ameliorates imiquimod-induced skin inflammation in a CD1A transgenic mouse model (36), indicating a role for CD1a-autoreactive $\mathrm{T}$ cells in promoting skin inflammation. However, the role of CD1b- and CD1c-autoreactive $\mathrm{T}$ cells in inflammatory diseases remains unknown.

Thus, to study the physiological relevance of autoreactive CD1b-restricted $\mathrm{T}$ cells in vivo, we used a double-transgenic mouse model that expresses human CD1b and CD1c molecules (37) and HJ1, a CD1b-autoreactive $\mathrm{T}$ cell receptor (hCD1Tg HJ1Tg mice) (38). Since the presence of these T cells did not lead to overt autoimmunity, we explored the role of CD1b-autoreactive $\mathrm{T}$ cells under conditions of hyperlipidemia by cross- 
A

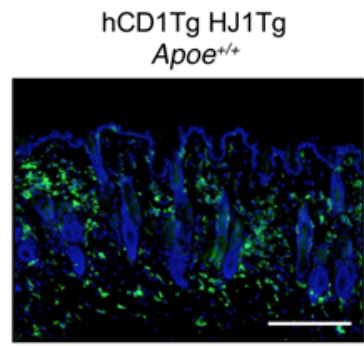

$\mathbf{B}$

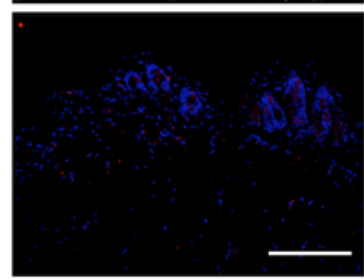

C

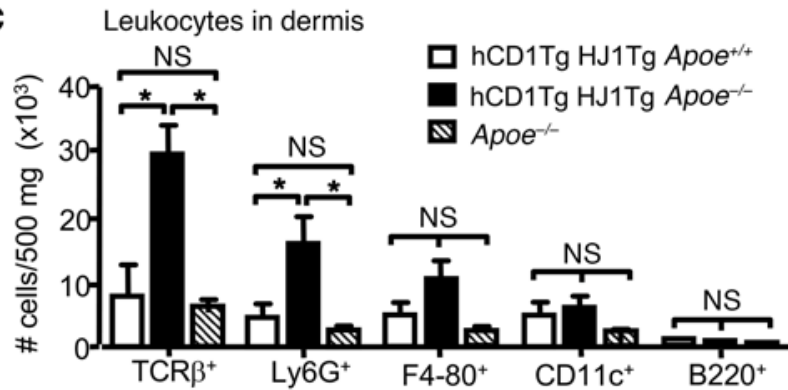

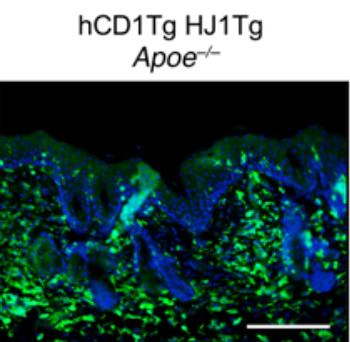
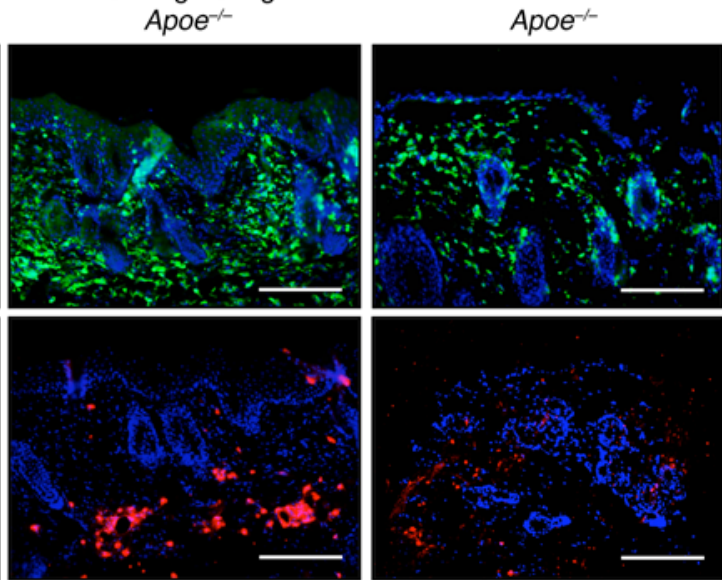

D Dermal HJ1 T cells (mRNA)

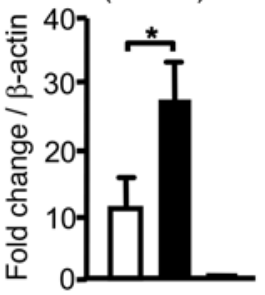

Anti CD3

(200x)

Anti-Gr-1

(200x)

Figure 2. Diseased skin in hCD1Tg $\mathrm{HJ} 1 \mathrm{Tg} \mathrm{Apoe}^{-/-}$mice is characterized by $T$ cell and neutrophil infiltrates. Indicated organs were harvested from mice at about 25 weeks of age when hCD1Tg HJ1Tg Apoe - $^{-/}$mice exhibited fulminant disease. (A and B) Immunofluorescence staining of skin sections from indicated mice with anti-CD3

(A) and anti-Gr-1 (B). Scale bars: 100 $\mu \mathrm{m}$. (C) Bar graph depicts the absolute number of various leukocyte subsets in the dermis of mice, performed using flow cytometry $(n=3-5)$. (D) mRNA analysis of HJ1 T cells in the skin of the mice using HJ1 TCR-specific primers. (E-G) hCD1Tg HJ1Tg Apoe - mice $^{-/}$have systemic neutrophil infiltration. Quantification of neutrophils (CD11b+Ly6C+cells) in the cervical LNs $(\mathbf{E})$, spleen (F), and liver (G) was performed using flow cytometry ( $n=4-6)$. (H) mRNA detected in the skin of different mouse strains using cytokinespecific primers $(n=3-5)$. mRNA levels were normalized relative to $\beta$-actin. Values are mean + SEM. ${ }^{* *} P<0.005$ ${ }^{*} P<0.01$. Statistical analyses were performed using 1-way ANOVA followed by Bonferroni's post-hoc test.

E

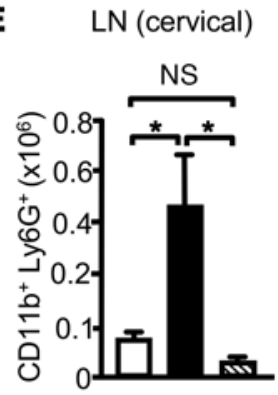

$\mathbf{F}$

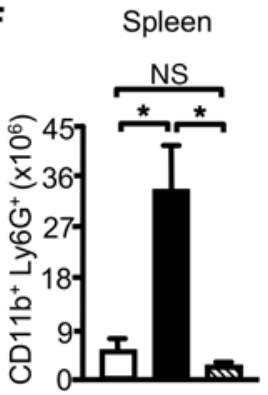

G

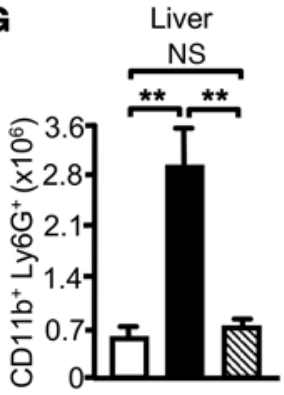

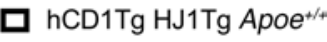

hCD1Tg HJ1Tg Apoe ${ }^{-/-}$

$A p o e^{-1-}$
H

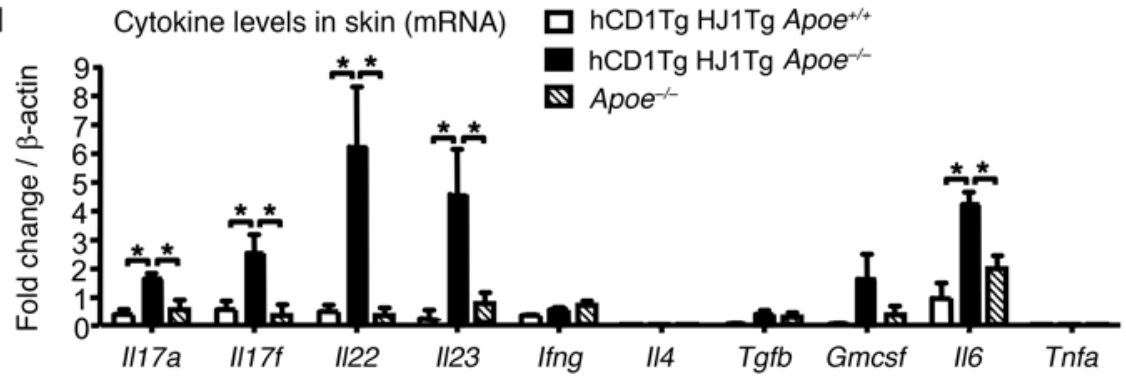

ing hCD1Tg HJ1Tg mice onto the ApoE-deficient background (hCD1Tg HJ1Tg Apoe ${ }^{-/}$). ApoE-deficient mice, which have elevated levels of serum triglyceride and cholesterol, provided a useful tool for studying the role of CD1b-autoreactive T cells in hyperlipidemia-associated inflammatory diseases, such as atherosclerosis and psoriasis.

In this study, we found that hCD1Tg HJ1Tg Apoe $e^{-/}$mice spontaneously developed psoriasis-like skin inflammation starting at
25 to 30 weeks of age. These mice also developed atherosclerotic lesions. Atherosclerotic plaque burden in hCD1Tg HJ1Tg Apoe -- $^{-}$ mice was comparable to that of $A p o e^{-/-}$mice at 30 weeks of age. HJ1 T cells in hCD1Tg HJ1Tg Apoe ${ }^{-/-}$mice were phenotypically more activated and produced elevated levels of IL-17A. Interestingly, an increased frequency of IL-17A-producing $\mathrm{T}$ cells was also detected in hCD1Tg Apoe $e^{-/-}$mice, which harbor polyclonal CD1b- and CD1c-restricted T cells. We found that hyperlipidemia 
enhanced IL- 6 secretion by CD1b ${ }^{+}$DCs and augmented IL-17A production by HJ1 T cells. Furthermore, phospholipid and cholesterol species, which preferentially accumulated in the diseased

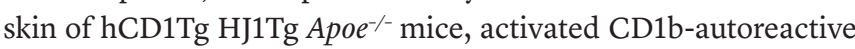
$\mathrm{HJ1}$ T cells. In parallel, we studied human patients and found that CD1b expression and CD1b-autoreactive T cells were increased in psoriatic individuals compared with healthy controls. Thus, this study demonstrates a link between hyperlipidemia and psoriasis, pointing to a specific and unexpected role of autoreactive CD1brestricted $\mathrm{T}$ cells in inflammatory skin diseases.

\section{Results}

hCD1Tg HJ1Tg Apoe-/- mice spontaneously develop psoriasiform dermatitis. hCD1Tg HJ1Tg mice, which expressed human CD1b and $\mathrm{CD} 1 \mathrm{c}$ as well as the CD1b-autoreactive $\mathrm{T}$ cell receptor (TCR) (HJ1) were crossed to the Apoe ${ }^{-/-}$background. hCD1Tg HJ1Tg Apoe $^{-/-}$mice progressively lost weight starting at about 16 weeks of age (Figure 1A). By 25 weeks of age, 95\% of hCD1Tg HJ1Tg Apoe ${ }^{-/-}$mice also developed severe dermatitis. This was in contrast to hCD1Tg HJ1Tg Apoe A $^{++}$and Apoe $e^{-/}$mice, neither of which lost weight or developed skin lesions at such a high frequency (Figure 1B). Interestingly, we also observed an increased incidence of dermatitis in hCD1Tg Apoe $e^{-/-}$mice ( $50 \%$ ) compared with Apoe ${ }^{-/-}$mice $(\sim 20 \%)$. This suggests that polyclonal autoreactive CD1b- and CD1c-restricted T cells naturally present in hCD1Tg Apoe $e^{-/}$mice may contribute to skin inflammation in response to hyperlipidemic conditions. Additionally, only $28 \%$ of HJ1Tg Apoe ${ }^{-/}$mice $\left(\mathrm{CD} 1 \mathrm{~b}^{-}\right.$and $\left.\mathrm{CD} 1 \mathrm{c}^{-}\right)$developed disease, suggesting that both CD1 and CD1-reactive $\mathrm{T}$ cells were required for high rates of disease development (Figure 1B). Skin sections obtained from diseased hCD1Tg HJ1Tg Apoe - $^{--}$mice showed epidermal hyperplasia (Figure 1B), increased leukocytic infiltration, and hyperkeratosis (Figure 1C), which are histologic features also found in psoriatic skin. Disease incidence was not different in male versus female mice. Keratinocytes, which are key effectors in the pathogenesis of psoriasis, proliferated more in the diseased skin, as indicated by Ki67 staining (Figure 1D).

Since the histopathology observed in diseased skin of hCD1Tg

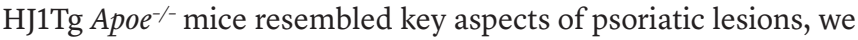
examined the levels of S100a proteins, which are known to be elevated in psoriasis patients. Indeed, $S 100 a 7$ and $S 100 a 8$ mRNA levels were higher in diseased hCD1Tg HJ1Tg ApoE $E^{-/-}$mice compared

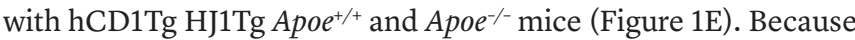
$A p o E^{-/-}$mice are characterized by hyperlipidemia and formation of atherosclerotic plaques, we also examined the effect of HJ1 T cells on plaque formation in the aortic root region. Upon staining of aortic root sections from hCD1Tg HJ1Tg Apoe - $^{--}$and Apoe $e^{-/}$mice with oil red $\mathrm{O}$, we observed plaque in both groups of animals at 30 weeks. However, there was no significant difference in plaque burden between these 2 mouse strains (Figure 1F). Additionally, we examined the colon of diseased hCD1Tg HJ1Tg Apoe ${ }^{-/}$mice for any obvious pathology by H\&E staining. As depicted in Supplemental Figure 1 (supplemental material available online with this article; https://oi.org/10.1172/JCI92217DS1), no significant differences were observed between hCD1Tg HJ1Tg Apoe $e^{+/+}$, hCD1Tg HJ1Tg Apoe $e^{-/}$, and Apoe $e^{-/-}$mice. Thus, Apoe deletion caused the expected development of atherosclerotic plaque, but we did not detect a measurable effect of HJ1 T cells on plaque formation at the time point examined. However, we found a marked, highly penetrant skin disease that was dependent on the presence of CD1b-autoreactive HJ1 T cells and the absence of ApoE.

Skin inflammation is characterized by $T$ cell and neutrophil infiltration along with a Th17-biased cytokine response. To characterize the leukocyte populations in the skin lesions, we stained skin sections from hCD1Tg HJ1Tg Apoe ${ }^{+/}$, hCD1Tg HJ1Tg Apoe ${ }^{-/}$, and Apoe $^{-/-}$mice with Abs against CD3 and Gr-1. Skin sections from

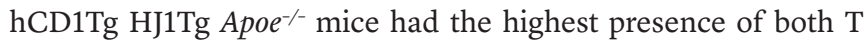
cells (Figure 2A) and $\mathrm{Gr}-1^{+}$granulocytes (Figure $2 \mathrm{~B}$ ), especially in the dermis. Flow cytometric analysis of skin cell suspensions confirmed an increased frequency of T cells and CD11 $\mathrm{b}^{+} \mathrm{Ly} 6 \mathrm{G}^{+}$neutrophils in hCD1Tg HJ1Tg Apoe -/ $^{-}$mice, while no significant differences in the percentages of B cells, macrophages, and DCs were detected among these 3 strains of mice (Figure 2C). To confirm that infiltrating $\mathrm{T}$ cells in diseased skin were indeed HJ1 T cells, HJ1 V $\beta$ chain-specific primers were used to detect the mRNA levels of these T cells. As expected, there were more HJ1 T cells in the skin of hCD1Tg HJ1Tg Apoe $e^{-/}$mice compared with hCD1Tg HJ1Tg $\mathrm{Apoe}^{+/+}$mice and no HJ1 T cells were detected in Apoe ${ }^{-/-}$mice (Figure 2D). Interestingly, increased numbers of neutrophils were also detected in the cervical LNs (Figure 2E), spleen (Figure 2F), and liver (Figure 2G) of hCD1Tg HJ1Tg Apoe ${ }^{-/-}$mice, demonstrating systemic inflammation at sites outside the skin.

To determine the cytokine milieu in the skin of diseased and healthy mice, mRNA levels of different cytokines were detected in hCD1Tg HJ1Tg Apoe $e^{+/}$, hCD1Tg HJ1Tg Apoe $e^{-/}$, and Apoe - $^{--}$mice. We found that the expression of Il6- and Th17-related cytokines, including Il17a, Il17f, Il22, and Il23, was substantially higher in the diseased hCD1Tg HJ1Tg Apoe $e^{-/}$mice compared with Apoe ${ }^{-/}$and hCD1Tg HJ1Tg Apoe ${ }^{+/+}$mice. However, there was no difference in Ifng, Il4, Tgfb, and GMCSF levels among the 3 groups (Figure $2 \mathrm{H}$ ). These data suggest that skin inflammation in hCD1Tg HJ1Tg Apoe $e^{-/-}$ mice was correlated with an increased Th17 response.

Dermal $\mathrm{T}$ cells in hCD1Tg HJ1Tg Apoe-- mice have a more activated phenotype and produce elevated levels of IL-17A. Since HJ1 T cells were markedly increased in the skin of hCD1Tg HJ1Tg Apoe-/mice, we next sought to compare the phenotype and function of these T cells with that of dermal T cells in hCD1Tg HJ1Tg Apoe $e^{+/+}$ and $A p o e^{-/-}$mice. Dermal T cells from hCD1Tg HJ1Tg Apoe $e^{-/-}$mice had the highest percentage of $\mathrm{CD} 69^{+} \mathrm{CD} 44^{+}$double-positive cells (Figure 3, A and B). A similar trend of $\mathrm{T}$ cell activation was also observed in the skin-draining cervical LNs (Figure 3, C and D). Interestingly, CD5 expression, a marker for $\mathrm{T}$ cell signaling strength, was highest in T cells isolated from hCD1Tg HJ1Tg Apoe $^{-/-}$mice (Figure 3, E and F), consistent with their heightened activation status. To determine whether an altered Treg population could contribute to the phenotype observed in hCD1Tg HJ1Tg Apoe $^{-/}$mice, we stained both dermal and cervical LN T cells for CD4 and FoxP3 to detect canonical Tregs. There was no difference in the $\mathrm{CD}^{+} \mathrm{FoxP}^{+}$population in either the cervical LNs (Supplemental Figure 2A) or the dermis (Supplemental Figure 2B) of hCD1Tg HJ1Tg Apoe ${ }^{+/+}$, hCD1Tg HJ1Tg Apoe ${ }^{-/}$, and Apoe $e^{-/-}$mice. Using phorbol 12-myristate 13-acetate (PMA) and ionomycin to evaluate function ex vivo, we found that $\mathrm{T}$ cells isolated from the skin (Figure 4, A and B) as well as cervical LNs (Figure 4, A and 
A

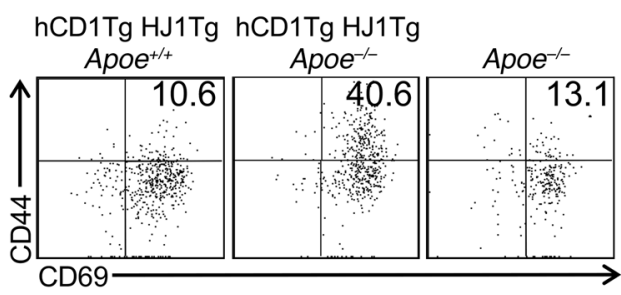

C

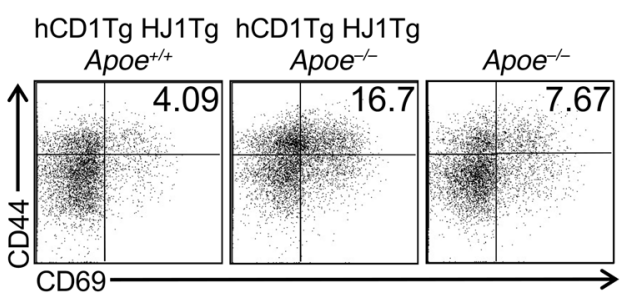

E

\section{hCD1Tg HJ1Tg hCD1Tg HJ1Tg}

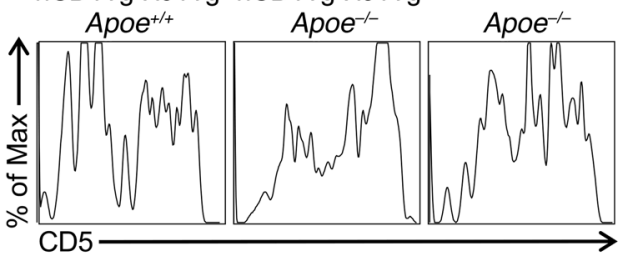

B

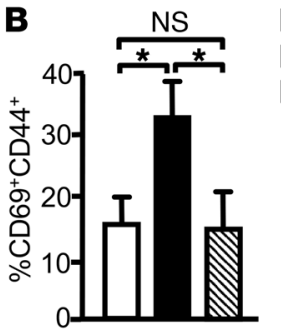

D
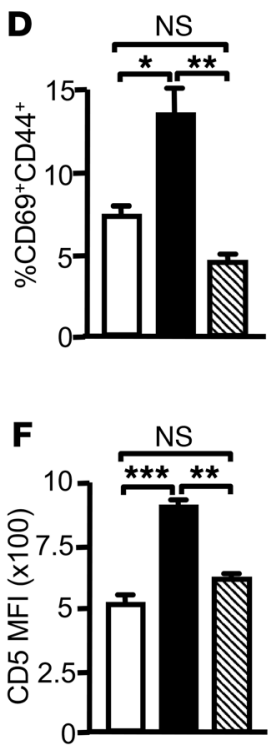

口 hCD1Tg HJ1Tg Apoe + +/

hCD1Tg HJ1Tg Apoe ${ }^{-/}$

$\mathrm{Apoe}^{-\mathrm{r}}$
Figure 3. Increased activation of $\mathrm{T}$ cells is observed in the skin and cervical LNs of hCD1Tg HJ1Tg Apoe $e^{-/-}$mice. Dermal and LN cells were isolated from indicated mice at 25 weeks of age, stained with anti-CD45 Abs, and gated on TCR $\beta^{+}$cells. Cell-surface staining was performed for T cell activation markers. (A and C) Representative FACS plots of the expression of $T$ cell activation markers CD69 and CD44 in the dermis (A) and cervical LNs (C). (B and D) Bar graphs depict the mean + SEM of the percentages of CD69+CD44+ T cells in the dermis (B) and LNs (D) of the mice $(n=5-10)$. (E) Representative FACS plots of CD5 expression on dermal $T$ cells from indicated mice. (F) Bar graph depicts the MFI of CD5 expression on dermal T cells in different mouse strains $(n=3) .{ }^{* *} P<0.005 ;{ }^{* *} P<0.01 ;{ }^{*} P<0.05$. Statistical analyses were performed using 1-way ANOVA followed by Bonferroni's post-hoc test.

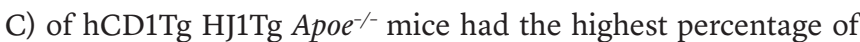
IL-17A-producing cells. However, no significant difference in the percentages of IFN- $\gamma$-producing $\mathrm{T}$ cells was detected in these 3 strains of mice (Figure 4, D-F). Overall, dermal T cells from diseased hCD1Tg HJ1Tg Apoe - $^{--}$mice were more activated and produced more IL-17A.

T cells in hCD1Tg HJ1Tg Apoe-/- and hCD1Tg Apoe-/- mice produce more IL-17A compared with their counterparts on an ApoE-sufficient background. To test whether enhanced Th17 response detected in hCD1Tg HJ1Tg Apoe ${ }^{-/}$mice reflected CD1b-dependent cytokine production by HJ1 T cells, purified T cells from either hCD1Tg HJ1Tg $\mathrm{Apoe}^{+/+}$or hCD1Tg HJ1Tg Apoe ${ }^{-/-}$mice were cocultured with WT $\left(\mathrm{CD}^{-} \mathrm{b}^{-}\right)$or hCD1Tg $\left(\mathrm{CD} 1 \mathrm{~b}^{+}\right) \mathrm{BM}$-derived DCs (BMDCs). Indeed, cytokine secretion was dependent on CD1b expression. hCD1Tg HJ1Tg Apoe $e^{--}$mice produced more IL-17A, but similar levels of IFN- $\gamma$, upon stimulation with hCD1Tg DCs compared with T cells from hCD1Tg HJ1Tg Apoe ${ }^{+/+}$mice (Figure 4G).

To determine whether enhanced IL-17A production by CD1bautoreactive HJ1 T cells in the context of ApoE deficiency could be detected at the polyclonal $\mathrm{T}$ cell level, $\mathrm{T}$ cells were isolated from hCD1Tg and hCD1Tg Apoe $e^{-/}$mice and incubated with either WT $\left(\mathrm{CD} 1 \mathrm{~b}^{-}\right)$or hCD1Tg (CD1b+) BMDCs. Interestingly, a higher frequency of polyclonal group 1 CD1-restricted IL-17A-producing

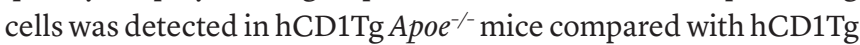
mice (Figure $4 \mathrm{H})$.

In vivo IL-17A neutralization ameliorates dermatitis. Since an elevated Th17 response was observed in diseased hCD1Tg HJ1Tg Apoe $^{-/-}$mice, we sought to determine whether systemic in vivo neutralization of IL-17A could ameliorate disease. Indeed, anti-IL17A treatment resulted in reduced leukocytic infiltration and epidermal hyperplasia in the skin of hCD1Tg HJ1Tg Apoe $e^{-/-}$mice, as shown by H\&E staining, compared with mice treated with isotype control Abs (Figure 5A). Flow cytometric analysis showed that the percentages of dermal neutrophils were decreased significantly in IL-17A-neutralized mice compared with isotype Ab-treated mice (Figure 5B). Dermal T cells from anti-IL-17A-treated hCD1Tg HJ1Tg Apoe ${ }^{-/}$mice had similar CD69 and CD44 expression profiles compared with healthy hCD1Tg HJ1Tg $\mathrm{Apoe}^{+/+}$mice (Figure 5C). Furthermore, IL-17A neutralization also led to decreased IL17A production by dermal T cells of hCD1Tg HJ1Tg Apoe ${ }^{-/-}$mice, but had no effect on IFN- $\gamma$ production (Figure $5, \mathrm{D}-\mathrm{F}$ ). These data indicated that IL-17A plays an important role in skin inflammation observed in hCD1Tg HJ1Tg Apoe $e^{-/-}$mice.

Hyperlipidemic serum enhanced IL-6 secretion by DCs and increased IL-17A production by HJ1 T cells. It has been demonstrated that hyperlipidemia can change DC maturation and thus function (39-41). To test the possibility that alterations in DC function can lead to the heightened production of IL-17A by HJ1 T cells, we isolated DCs from the LNs of hCD1Tg, hCD1Tg Apoe $e^{-/}$, and Apoe $e^{-/-}$ mice and incubated them with either normal mouse serum from C57BL/6 mice or hyperlipidemic serum from $\mathrm{Apoe}^{-/-}$mice. Interestingly, incubation of all 3 DC types in hyperlipidemic serum resulted in increased IL-6 production (Figure 6A). Since IL-6 can promote the production of Th17-type cytokines, we cocultured HJ1 T cells from hCD1Tg HJ1Tg Rag-/- mice with DCs from hCD1Tg, hCD1Tg $A p o e^{-/-}$, and Apoe $e^{-/-}$mice in normal and hyperlipidemic 
A

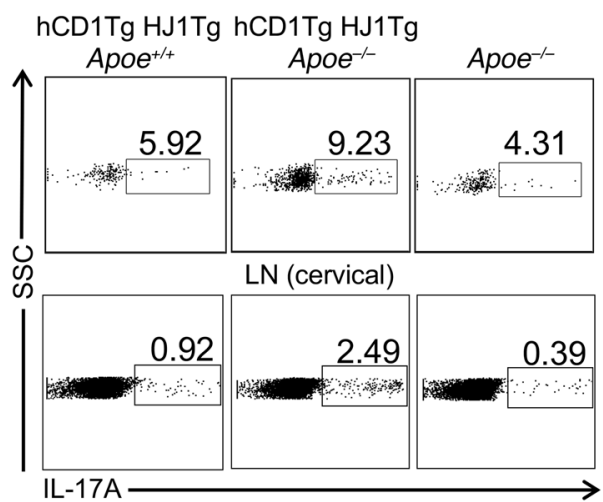

D

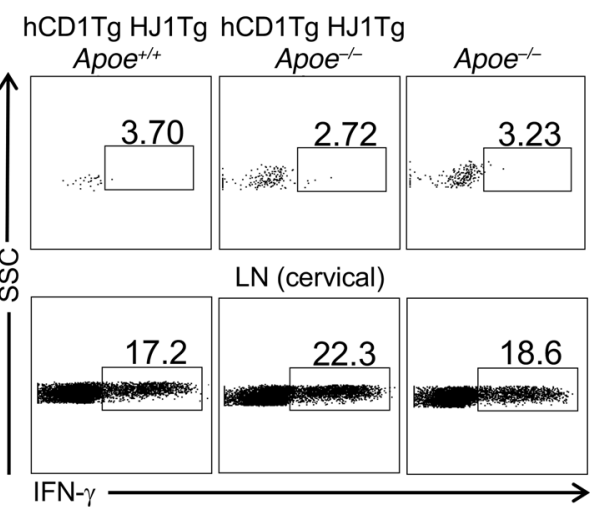

B

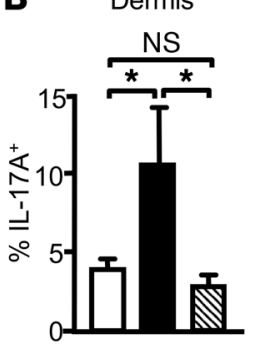

C $\quad \mathrm{LN}$ (cervical)

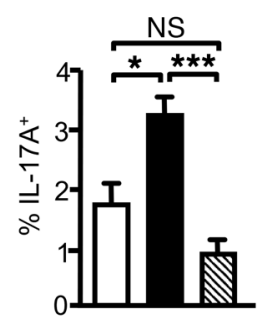

口 hCD1Tg HJ1Tg Apoe A $^{++}$

hCD1Tg HJ1Tg Apoe - $^{-/}$

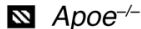

E

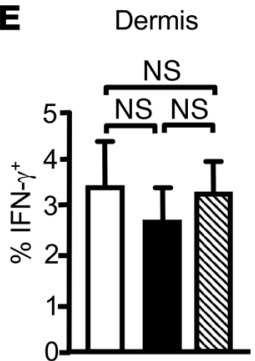

F $\quad$ LN (cervical)

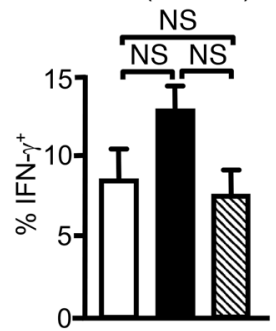

Figure 4. T cells in hCD1Tg HJ1Tg Apoe A $^{-/}$ mice produce elevated levels of IL-17A. Dermal and LN cells from indicated mice were stimulated with PMA/ionomycin to measure intracellular cytokine secretion. Cells were gated on $\mathrm{CD} 45^{+}$and TCR $\beta^{+}$populations. (A) Representative FACS plots of IL-17A-producing $T$ cells in the dermis (upper panels) and cervical LNs (lower panels) in indicated mice. (B and C) Bar graphs (mean + SEM) depict the percentages of IL-17A-producing $T$ cells in the dermis (B) and cervical LNs (C). (D) Representative FACS plots of IFN- $\gamma$-producing $T$ cells in the dermis and cervical LNs. (E and F) Quantification of IFN- $\gamma$-producing $T$ cells in the dermis (E) and cervical LNs (F) $(n=4-5)$. (G) WT (hCD1Tg') and hCD1Tg ${ }^{+}$ BMDCs were cocultured with enriched T cells from either hCD1Tg HJ1Tg Apoe (t+ $^{\text {or hCD1Tg }}$ HJ1Tg Apoe $e^{-/-}$mice for 48 hours. Amount of IL-17A (left panel) and IFN- $\gamma$ (right panel) was determined by ELISA. Data are representative of 3 independent experiments. (H) T cells were enriched from hCD1Tg and hCD1Tg $\mathrm{Apoe}^{-/-}$mice and cocultured with WT or hCD1Tg+ DCs for 24 hours. IL-17A-producing cells were quantified by ELISPOT assays. Data are representative of 2 independent experiments. ${ }^{* *} P<0.005$; ${ }^{* *} P<0.01$; ${ }^{*} P<0.05$. Statistical analyses were performed using 1-way ANOVA followed by Bonferroni's post-hoc test for 3-group comparisons and Student's $t$ test for 2-group comparisons.

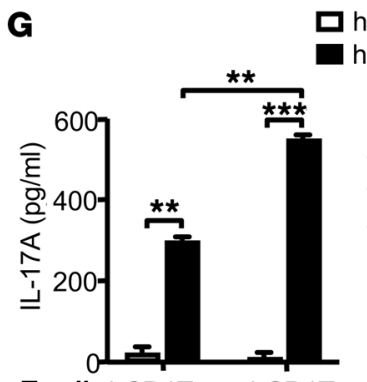

T cell hCD1Tg hCD1Tg source HJ1Tg HJ1Tg Apoe--

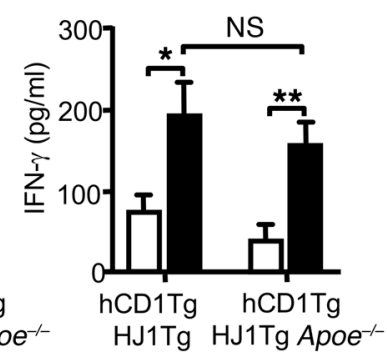

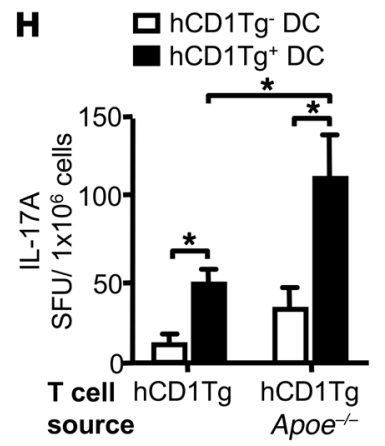

serum. Indeed, IL-17A production was the highest in the presence of hyperlipidemic serum with either hCD1Tg or hCD1Tg Apoe DCs (Figure 6B). A low level of IL-17A was detected when HJ1 T cells were cultured with $A$ poe $e^{-/}$DCs $\left(\mathrm{CD}^{-} \mathrm{b}^{-}\right)$in hyperlipidemic serum, suggesting that elevated levels of cytokines such as IL-6 may activate $\mathrm{HJ} 1 \mathrm{~T}$ cells in a CD1b-independent manner. In contrast, there was no significant difference in the amount of IFN- $\gamma$ present in the cocultures under different conditions and DC types (Figure 6B). These data suggest that hyperlipidemic serum could alter DC function, polarizing HJ1 T cells.

The expression of costimulatory molecule CD86 on CD1bexpressing DCs is upregulated in hCD1Tg HJ1Tg Apoe - $^{-/}$mice. It has been demonstrated that the expression pattern of group 1 CD1 molecules in hCD1Tg mice closely resembles that of humans (37). Indeed, CD1b expression in the skin of hCD1Tg mice is confined to dermal DCs, as typically seen in normal human skin (24). On the other hand, cytokine (42) or bacterially induced inflammation $(43,44)$ can promote group 1 CD1 expression on cells that do not normally express CD1 proteins. To explore which DC subsets expressed CD1b in inflamed transgenic mouse skin and evaluate their maturation status, cells from the dermis of hCD1Tg HJ1Tg Apoe $^{+/+}$, hCD1Tg HJ1Tg Apoe $e^{--}$, and Apoe $e^{-/}$mice were stained with various surface markers and subjected to FACS analysis. CD1b expression was mostly detected on $\mathrm{CD} 11 \mathrm{~b}^{+} \mathrm{CD} 11 \mathrm{c}^{+}$dermal DCs (Supplemental Figure 3A). However, there were no detectable differences in the percentages and total numbers of CD1bexpressing cells between diseased and nondiseased mice (Figure 6, C and D). To verify that there was no CD1b expression on Langerhans cells, which express high levels of CD1a, in the epidermis, epidermal cells were stained with CD11c and CD207. However, no CD1b expression was detected on Langerhans cells even in diseased hCD1Tg HJ1Tg Apoe $e^{-/}$mice (Supplemental Figure 3B). 
A

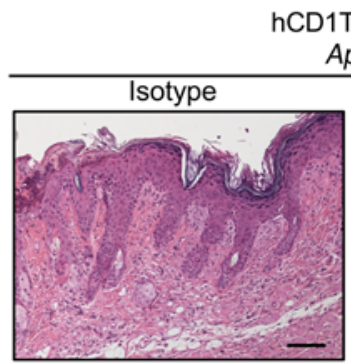

\section{B}
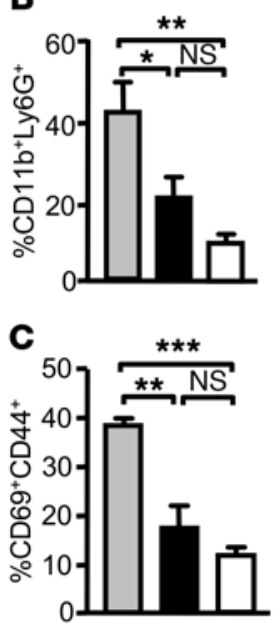

hCD1Tg HJ1Tg Apoe $^{-/-}$

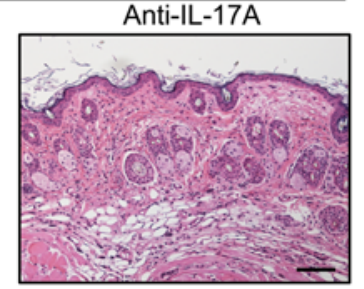

口 Isotype

Anti-IL-17A

口 $\mathrm{hCD} 1 \mathrm{Tg}$ HJ1Tg Apoe $^{+/+}$

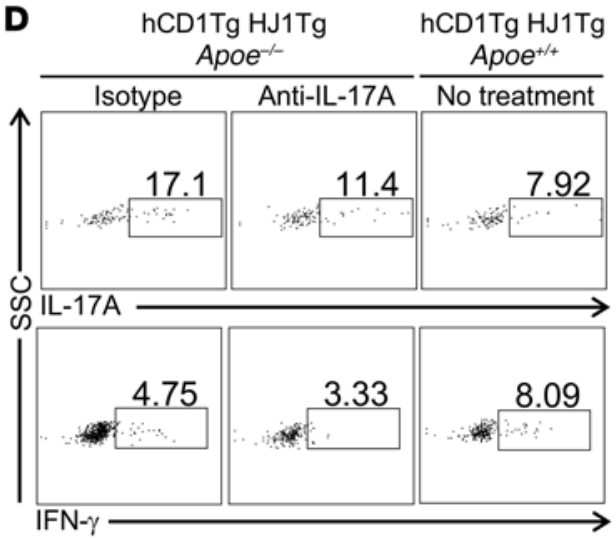

hCD1Tg HJ1Tg

Apoe $^{+/+}$

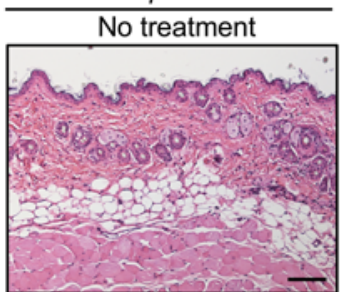

E

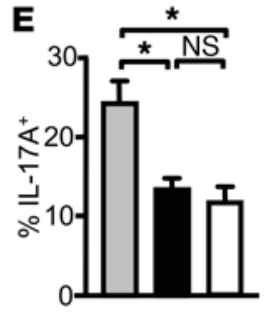

F

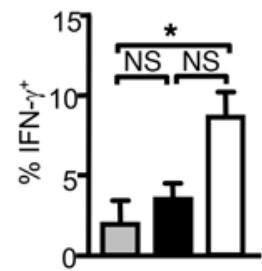

Figure 5. In vivo anti-IL-17A treatment ameliorates skin inflammation in hCD1Tg HJ1Tg Apoe ${ }^{-/-}$mice. (A) Representative H\&E staining of skin sections from untreated hCD1Tg/HJ1Tg mice and hCD1Tg HJ1Tg Apoe ${ }^{-/}$ mice treated with either anti-IL-17A mAbs or isotype control Abs. Scale bars: $100 \mu \mathrm{m}$. Cells from the dermis of mice were isolated, stained with indicated Abs, and analyzed by flow cytometry. (B) Bar graph depicts the percentages of neutrophils (CD11 b+Ly6 $\mathrm{C}^{+}$) within the $\mathrm{CD}_{4} 5^{+}$gated population from different groups of mice. (C) Bar graph depicts the percentages of $C D 69^{+} \mathrm{CD} 44^{+}$cells within the TCR $\beta^{+} C D 45^{+}$gated population from different groups of mice. (D-F) Intracellular cytokine production was measured in dermal T cells after PMA/ionoycin stimulation. (D) Representative FACS plots of IL-17Aproducing (upper panels) and IFN- $\gamma$-producing (lower panels) T cells in the skin of indicated mice. (E and F) Bar graphs (mean + SEM) depict the percentages of IL-17A(E) and IFN- $\gamma$-producing (F) T cells in the skin of 3 different groups of mice $(n=3$ per group). ${ }^{* *} P<0.005 ;{ }^{* *} P<0.01$; ${ }^{*} P<0.05$. Statistical analyses were performed using 1-way ANOVA followed by Bonferroni's post-hoc test.
Interestingly, CD86 expression on $\mathrm{CD} 1 \mathrm{~b}^{+} \mathrm{DCs}$ was increased in diseased hCD1Tg HJ1Tg Apoe ${ }^{-/-}$mice (Figure 6E). This suggested that CD1b-expressing DCs were more mature in diseased mice, possibly aiding the priming of HJ1 T cells in the skin. Furthermore, to investigate the possibility that skin disease might induce CD1b expression on nonhematopoietic cells, hair follicle stem cells $\left(\mathrm{CD} 45^{-}\right.$, integrin $\alpha_{6}^{+}$, integrin $\left.\beta_{1}^{+}, \mathrm{CD} 34^{+}, \mathrm{Sca}-1^{-}\right)$and keratinocytes $\left(\mathrm{CD} 45^{-}\right.$, integrin $\alpha_{6}^{+}$, integrin $\left.\beta_{1}^{+}, \mathrm{CD} 34^{-}, \mathrm{Sca}-1^{+}\right)$ from the epidermis of hCD1Tg HJ1Tg Apoe ${ }^{+/+}$, hCD1Tg HJ1Tg $\mathrm{Apoe}^{-/}$, and $\mathrm{Apoe}^{-/-}$mice were stained with anti-CD1b. We found no appreciable staining on either epidermal keratinocytes or hair follicle stem cells, even in diseased hCD1Tg HJ1Tg Apo $e^{-/-}$mice (Supplemental Figure 3C).

Phospholipids and cholesterol preferentially accumulate in the skin of hCD1Tg HJ1Tg Apoe ${ }^{-/-}$mice. To explore the possibility that alteration of lipid composition and quantity in the skin of

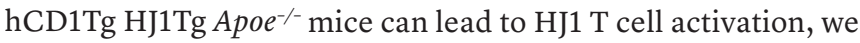
first determined the lipid profiles of diseased and normal mice. Total lipid was extracted from the skin and liver of hCD1Tg

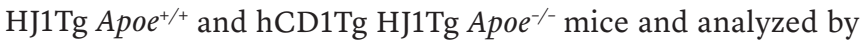
mass spectrometry or gas chromatography. We found that phospholipid species were present in much higher levels in the skin

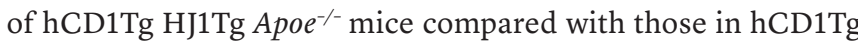

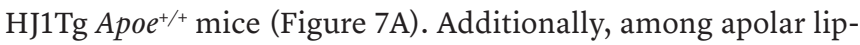
ids tested, cholesterol, cholesterol esters, and ceramides accumulated in diseased skin of hCD1Tg HJ1Tg Apoe ${ }^{-/-}$mice (Figure 7B). Furthermore, the ratio of phospholipids (Figure 7C) and cholesterol (Figure 7D) between diseased and healthy mice was substantially higher in the skin compared with the liver, an organ that is involved in lipid metabolism. This suggested that
ApoE deficiency led to the preferential accumulation of lipids in the skin. Interestingly, shorter chain fatty acids were more prevalent in healthy skin, while longer chain fatty acids were present at higher quantities in diseased skin. However, there was no difference in fatty acid accumulation in the skin versus the liver (Supplemental Figure 4, A and B).

Recent studies that identified lipid autoantigens in vitro pointed to candidate self-lipids that might function in this in vivo disease, including cholesterol (31), fatty acids (17), and membrane phospholipids $(19,45)$. To identify self-lipids presented by CD1b to HJ1 T cells, CD1b plate-bound assays were performed. In a previous study, we have shown that HJ1 T cells cannot be activated by different species of ceramides (46). Commercially purchased polar lipid extract, cholesterol, and fatty acid mixtures were loaded onto purified CD1b protein, coated onto 96-well plates, and cultured with HJ1 hybridoma cells. IL-2 secretion was measured as a read-out for HJ1 T cell activation. Polar lipid extract (comprising mostly phospholipids) stimulated HJ1 T cell hybridoma most strongly; cholesterol also activated the hybridoma cells, but to a lesser extent compared with polar lipids. However, neither saturated plus monounsaturated fatty acids (FA1) nor polyunsaturated (FA2) fatty acids had any HJ1 T cell stimulatory capacity (Figure 7E). This suggested that CD1b could present some lipids that preferentially accumulated in the skin of hCD1Tg HJ1Tg Apoe $e^{-/-}$mice and activated HJ1 T cells. Additionally, another CD1b-autoreactive mouse T cell hybridoma, LN1-7, showed reactivity similar to that of HJ1. While LN1-7 responded more strongly to polar lipids, they were less potently activated by cholesterol compared with HJ1 hybridoma cells (Supplemental Figure 4C). 
A

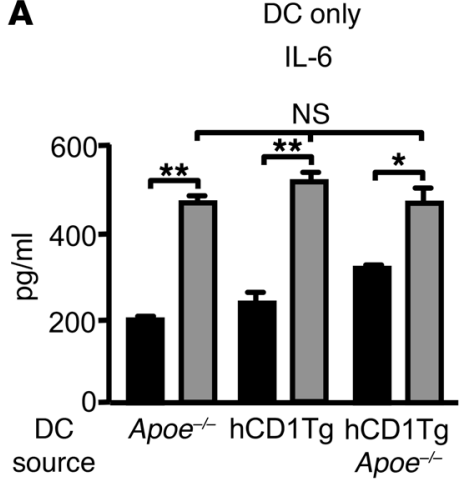

B

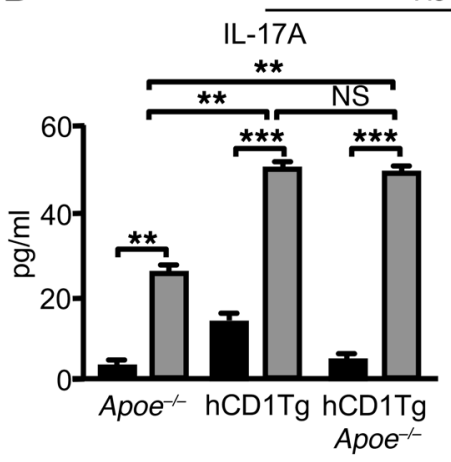

HJ1 T + DC

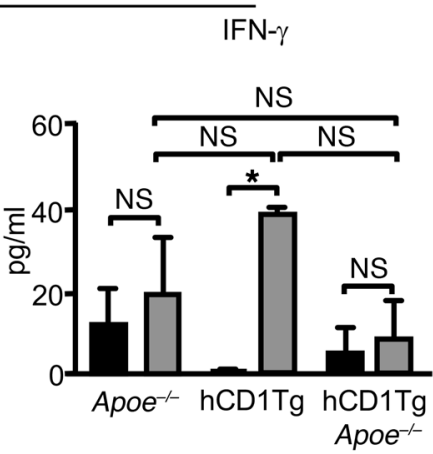

Apoe $^{+}$serum

$A p o e^{-/}$serum
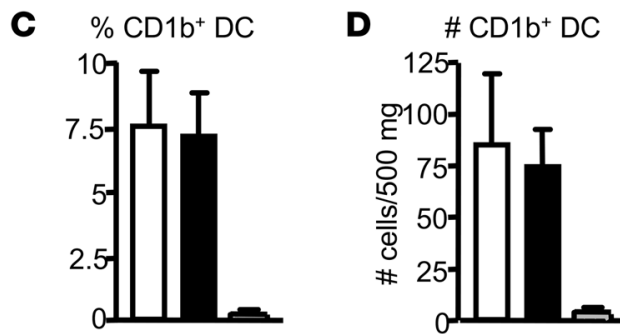

$\square$ hCD1Tg HJ1Tg Apoe
' hCD1Tg HJ1Tg Apoe
$\square$ Apoe $^{-/ /}$
E

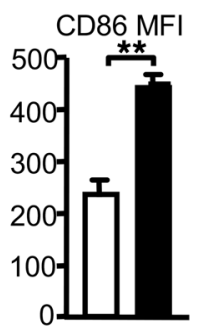

口 hCD1Tg HJ1Tg Apoe ${ }^{+/+}$

hCD1Tg HJ1Tg Apoe-

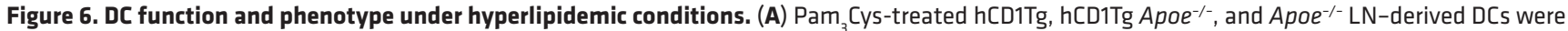
cultured in $\mathrm{Apoe}^{+/+}$and $A p o e^{-/-}$mouse serum. IL-6 was measured by cytometric bead array (CBA) after 48 hours. (B) hCD1Tg, hCD1Tg Apoe ${ }^{-/,}$, and Apoe ${ }^{-/-}$

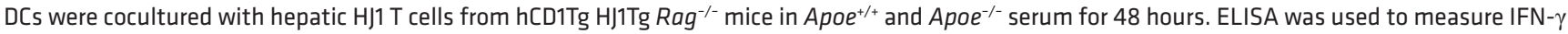
and IL-17A secretion. Data are representative of at least 2 independent experiments. (C-E) Cells from the dermis were isolated, stained with various Abs, and gated on CD45 and CD11 b+CD11 ${ }^{+}$DCs. Percentages (C) and numbers (D) of CD1b-positive cells in the skin were quantified. (E) CD1b+DCs (gated on CD11 ${ }^{+}$CD11C ${ }^{+}$population) were examined for their expression of costimulatory molecule CD86 $(n=4) .{ }^{* *} P<0.005 ;{ }^{* *} P<0.01$; ${ }^{*} P<0.05$. Statistical analyses were performed using 1-way ANOVA followed by Bonferroni's post-hoc test for 3 group comparisons and Student's $t$ test for 2 group comparisons.

Next, we considered whether human T cells exist that have similar molecular targets compared with HJ1 T cells. Previously published screens of peripheral blood lymphocytes have found higher rates of autoreactive response to CD1a and CD1c as compared with CD1b $(16,18)$, so evidence for CD1b autoreactivity is limited (26). However, a recent study used human CD1b tetramers to identify $\mathrm{T}$ cell lines and clones, including A25Salmonella (A25) and BC8Staphylococcus (BC8), which were autoreactive to CD1b and specifically recognized membrane phospholipids (19). This recent work provides clear evidence for the existence of $\mathrm{T}$ cell autoreactivity to CD1b and phospholipids in humans. Based on this information, we loaded phospholipid antigens identified in the HJ1 system, including phosphatidylglycerol (PG) and phosphatidylethanolamine (PE), onto human CD1b tetramers. We observed bright, lipid-dependent staining of A25 and BC8 (Figure 7F), confirming that antigens for the HJ1 TCR are recognized by other TCRs from human blood.

$C D 1 b$-autoreactive $T$ cell frequency is increased in patients with psoriasis. To determine whether group $1 \mathrm{CD} 1$ expression in skin from psoriatic patients with hyperlipidemia was altered compared with normal skin, we stained skin sections with mAbs against CD1a, CD1b, and CD1c. As expected, compared with anti-CD1b and antiCD1c, anti-CD1a stained the largest number of cells in control skin, which are likely Langerhans cells. The number of cells stained for
CD1b was dramatically increased in psoriasis patients, an effect that was most clearly seen in the dermis and was not seen for CD1a or CD1c (Figure 8, A and B). To evaluate group 1 CD1-restricted $\mathrm{T}$ cell frequency and function in psoriatic patients, blood samples were collected from psoriasis patients and healthy donors. A significantly higher frequency of CD1a- and CD1b-reactive T cells was detected in the peripheral blood mononuclear cells (PBMCs) of psoriatic patients compared with healthy individuals (Figure $8 \mathrm{C}$ ). However, CD1c-autoreactive T cells could not be detected in either psoriatic or healthy individuals (Figure 8C).

To compare the frequency of CD1b-autoreactive $\mathrm{T}$ cells in the peripheral blood of normal and psoriatic patients, we took advantage of recently validated tetramers that were untreated or loaded with known phospholipid autoantigens PG and PE (19). It is known that untreated CD1b tetramers harbor endogenous lipid antigens (19); thus, it is not surprising that some CD1b-autoreactive T cells were detected by untreated CD1b tetramers. Psoriatic patients had statistically higher percentages of CD1b tetramerpositive T cells compared with healthy controls (Figure 8, D and E). Thus, our study demonstrated that group 1 CD1-restricted $\mathrm{T}$ cells not only play a role in our mouse model of hyperlipidemiainduced spontaneous skin inflammation, but also are increased in frequency in human psoriatic patients. 
A Skin polar lipids

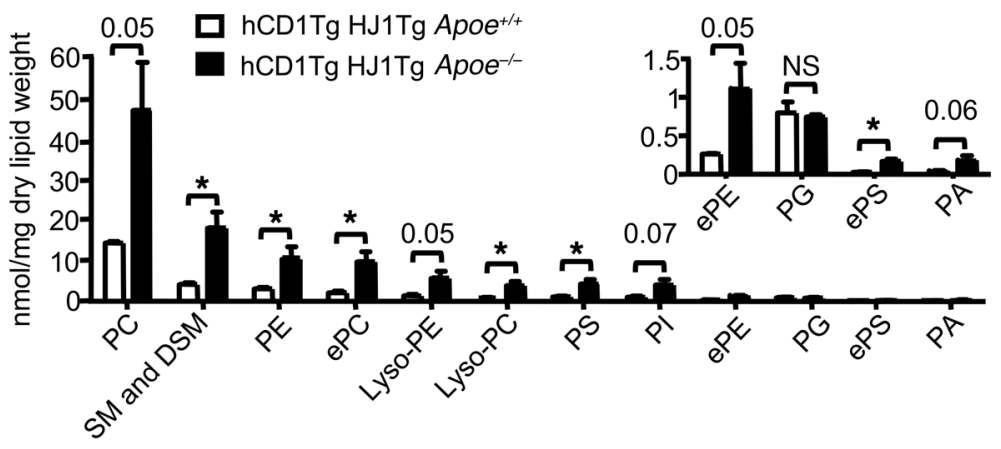

B Fold change

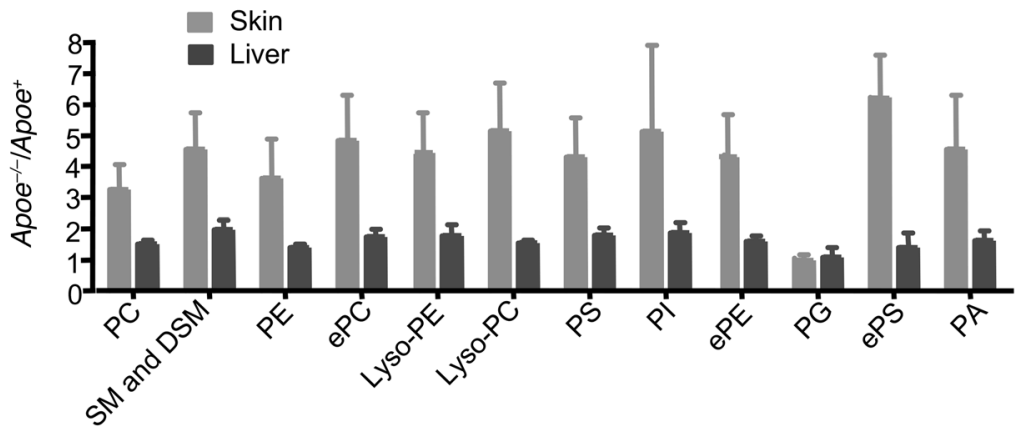

C Skin sterols

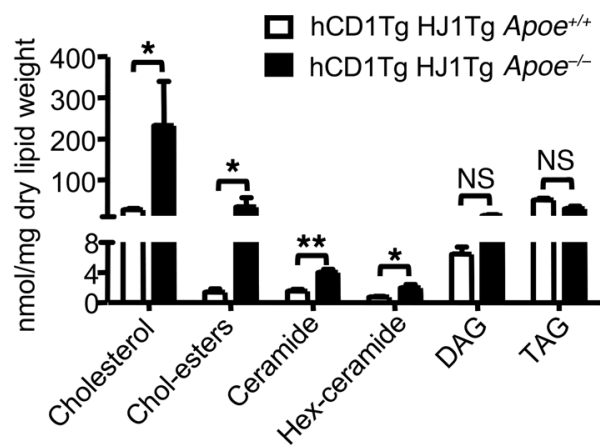

D Fold change

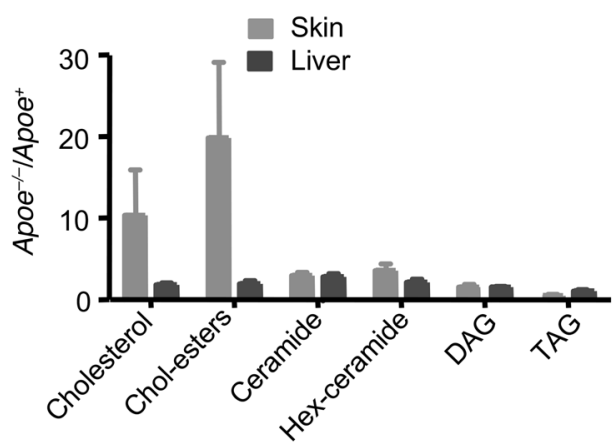

E HJ1 reactivity to lipids

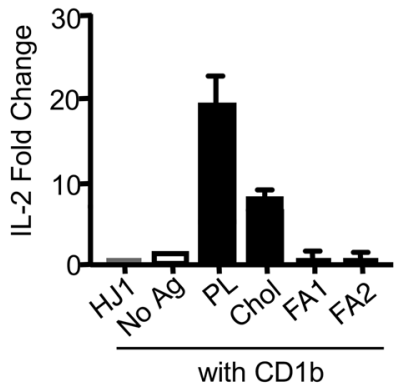

F CD1b-autoreactive human T cell clones

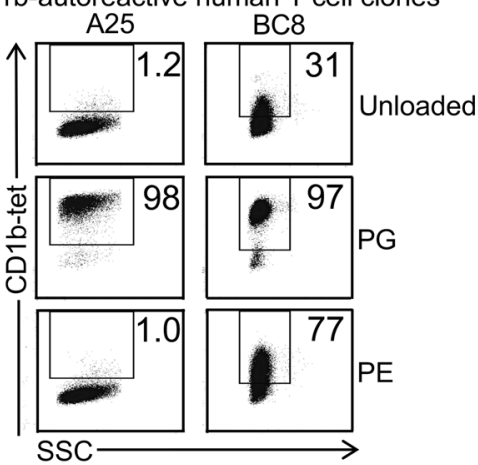

Figure 7. Phospholipid and cholesterol species, which preferentially accumulate in the skin of hCD1Tg HJ1Tg Apoe ${ }^{-/-}$mice, can activate HJ1 T cells. Total lipid was extracted from skin and liver tissues of mice, weighed, and analyzed by mass spectrometry and gas chromatography. (A) Comparison of phospholipid accumulation in the skin of diseased hCD1Tg HJ1Tg Apoe $e^{-/-}$and healthy hCD1Tg HJ1Tg Apoe ${ }^{+/+}$mice. (B) Ratio of phospholipids from diseased over healthy mice in the skin and liver. PC, phosphatidylcholine; SM, sphingomyelin; DSM, dihydro-sphingomyelin; ePC, ether-linked phosphatidylcholine; PS, phosphatidylserine; PI, phosphatidylinositol; ePE, ether-linked phosphatidylethanolamine; ePS, ether-linked phosphatidylserine; PA, phosphatidic acid. (C) Comparison of apolar lipid accumulation in the skin of diseased hCD1Tg HJ1Tg Apoe ${ }^{-/-}$and healthy hCD1Tg HJ1Tg Apoe ${ }^{+/+}$mice. (D) Ratio of apolar lipids from diseased over healthy mice in the skin and liver $(n=3)$. (E) Polar lipid extract (PL), cholesterol (Chol), and fatty acid mixtures (FA1 and FA2) were loaded onto CD1b protein and incubated with HJ1 T cell hybridoma for 24 hours. IL-2 in the supernatant was measured by ELISA. (F) CD1b-autoreactive human T cell clones were stained with mock-loaded CD1b tetramers or PG- or PE-loaded CD1b tetramers. Data are representative of at least 3 experiments. ${ }^{* *} P<0.005$; ${ }^{* *} P<0.01 ;{ }^{*} P<0.05$, Student's $t$ test.

\section{Discussion}

Using a transgenic mouse model expressing human CD1b and CD1c, we observed severe, highly penetrant skin disease that appears when ApoE is absent and when CD1b and CD1b-autoreactive $\mathrm{T}$ cells are present. Further, we showed that skin, which is disproportionally a site of lipid accumulation, is also the site where substantial inflammation developed starting at 25 weeks of age. In contrast, no apparent pathology was observed in the colon (Supplemental Figure 1), kidneys, and pancreas of these mice (data not shown). Our data also demonstrate that CD1b-expressing DCs mediated a $\mathrm{T}$ cell response and provided a specific basis for candidate mechanisms by which hyperlipidemia lead to lipid accumulation in skin, expression of CD1 proteins, and presentation of selflipids to CD1b-autoreactive T cells, leading to a Th17 phenotype. The skin inflammation observed here was characterized by $\mathrm{T}$ cell infiltration as well as typical secondary features of Th17 inflammation, including granulocytosis. Finally, the syndrome was substantially reversed by anti-IL-17A, demonstrating a critical role of IL-17 
A
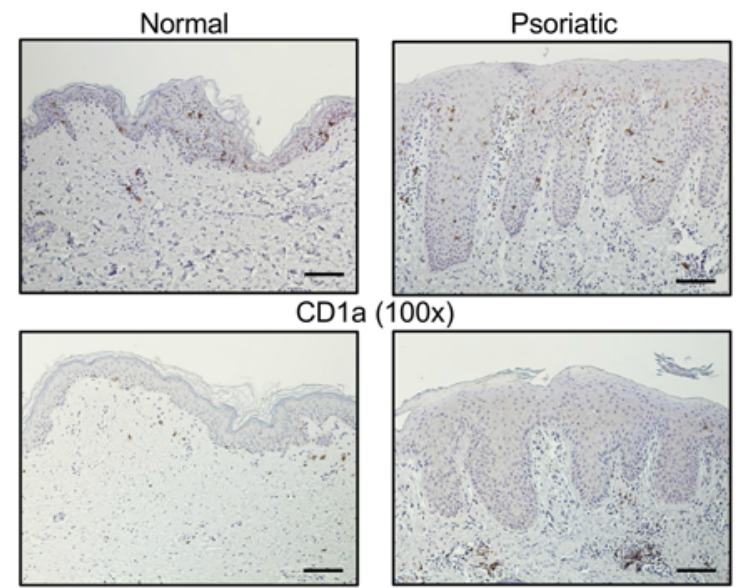

CD1b (100x)

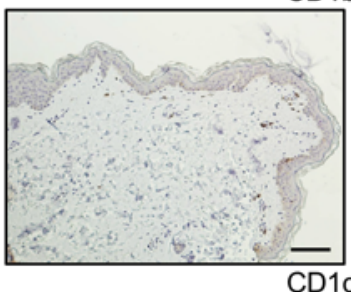

CD1c (100x)

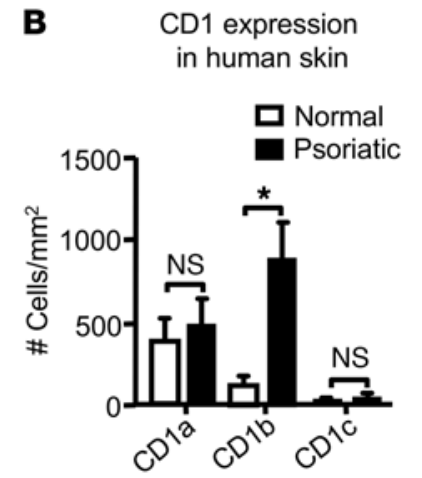

D
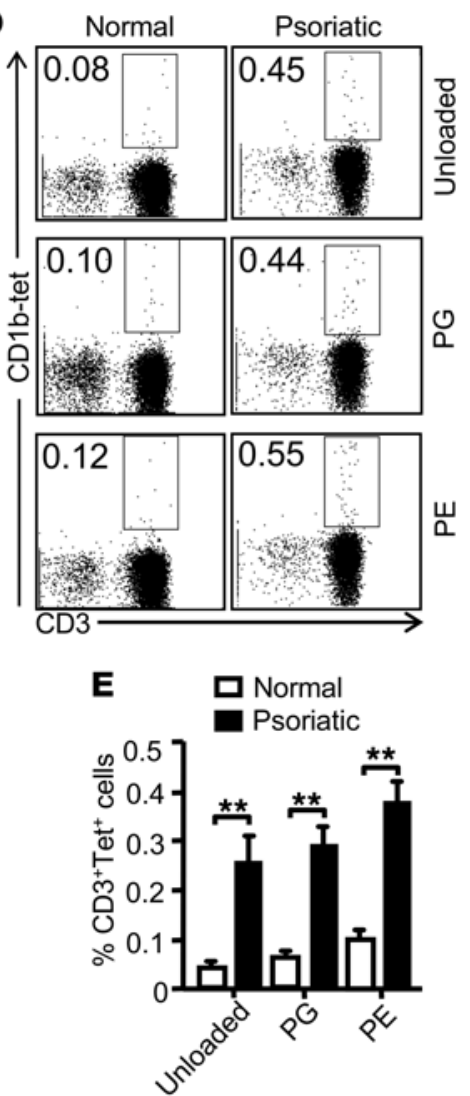

Figure 8. Psoriatic patients have an increased frequency of autoreactive group 1 CD1-restricted T cells. Psoriatic and normal skin biopsies were stained with Abs against CD1a, CD1b, and CD1c. Scale bars: 100 $\mu \mathrm{m}$. (B) Bar graph depicts number of CD1a-, CD1b-, and CD1c-positive cells $/ \mathrm{mm}^{2}$ (mean + SEM) $(n=3)$; skin biopsies were derived from psoriatic patients who were hyperlipidemic. (C) K562 alone and K562 CD1 transfectants were cocultured with human T cells $(n=9)$ that were stimulated twice with autologous mo-DCs followed by IL-2 ELISPOT assay. (D) PBMCs from psoriatic and normal individuals were stained with untreated CD1b tetramers or CD1b tetramers loaded with PE and PG ( $n=5$ for psoriatic patients; $n=8$ for normal individuals). Cells depicted on the FACS plots were first gated on the lymphocyte population and then on CD19- cells. (E) Bar graph depicts mean \pm SEM of the percentages of tetramer-positive cells. ${ }^{* * *} P<0.005$; ${ }^{* *} P<0.01$; ${ }^{*} P<0.05$, Mann-Whitney $U$ test. in this skin disease. Thus, these data unequivocally establish a new mouse model of lipid- and CD1b-driven T cell response that causes an autoimmune skin disease and signs of systemic neutrophilia. We believe these findings are among the first to demonstrate a specific role for human CD1b in inflammatory disease in vivo.

Unlike humans, commonly used laboratory animal models such as mice do not develop psoriasis-like skin inflammation. Therefore, numerous mouse models for studying psoriasis have been developed by genetic engineering, expressing psoriasis susceptibility genes on a transgene, xenografting human skin onto immunodeficient mice, or applying skin irritants $(47,48)$. However, spontaneously occurring psoriasis-like skin inflammation syndromes in mice are rare. This study describes a spontaneous and robust model of disease that not only recapitulates key features of psoriasis-like hyperkeratosis, parakeratosis, epidermal hyperplasia, and $\mathrm{T}$ cell and neutrophil infiltration, but also unearths an important role for $\alpha \beta$ self-lipid-specific T cells in skin inflammation upon conditions of hyperlipidemia. A major limitation of existing mouse models of $\mathrm{T}$ cell-mediated skin dis- ease is that mouse skin has abundant populations of $\gamma \delta \mathrm{T}$ cells known as dendritic epidermal $\mathrm{T}$ cells (DETC), which have no counterpart in humans (49). Conversely, normal human skin is dominated by $\alpha \beta \mathrm{T}$ cells, including those that recognize human targets, including CD1a, CD1b, and CD1c. Here we have identified a new in vivo dermatitis model that arises due to transfer of key parts of the human immune response to mice, specifically $\mathrm{CD} 1 \mathrm{~b}$ proteins and the responding $\alpha \beta \mathrm{T}$ cells.

Human epidemiological studies have identified links among psoriasis, hyperlipidemia, and atherosclerotic heart disease, but the precise causal nature of these interrelated factors is not well established $(3,5,6)$. For example, psoriasis patients are known to have increased plasma lipid levels (4). Most models of pathogenesis posit that dermal and systemic inflammation are the causes of skin changes and that hyperlipidemia, along with inflammation, promotes atherosclerosis. This syndrome appears after chronic, organ-specific lipid accumulation and requires a lipid-specific $\mathrm{T}$ cell response. Thus, these data potentially support new views about the complex relationships among hyperlipidemia, inflam- 
mation, and organ damage. Even though almost all of the hCD1Tg $\mathrm{HJ1Tg} \mathrm{Apoe}^{-/-}$mice developed psoriasiform dermatitis and aortic root plaques, plaque size was not different from that of $A p o e^{-/-}$ mice. This suggests that the presence of the CD1b-autoreactive HJ1 T cells does not detectably aggravate atherosclerotic plaque formation, at least at the time point examined. Whether HJ1 T cells might play an important role in plaque formation at later time points could not be determined, as mice needed to be euthanized by about 30 weeks of age due to weight loss and skin inflammation. Given the strong influence of invariant NKT (iNKT) cells in several models of atherosclerosis (50-53), our finding that HJ1 $\mathrm{T}$ cells do not affect plaque formation was initially surprising. It can be speculated that such differences may arise due to functional disparities between iNKT cells and HJ1 T cells, such as the strong Th17 polarization observed for HJ1 cells as well as tissuespecific differences in the location of lipid deposits and expression of CD1d and CD1b proteins. In addition, our mice showed weight loss, so that their depleted nutritional status might have partially reversed the effects of ApoE deletion. While iNKT cells are known to secrete IFN- $\gamma$ during atherosclerosis, $\mathrm{HJ} 1 \mathrm{~T}$ cells predominantly secrete IL-17A in hCD1Tg HJ1Tg Apoe -- $^{-}$mice. Interestingly, IL-17A has been known to impart both atherogenic and antiatherogenic effects on plaque formation (54).

Although psoriasis is a complex disease of unknown causes, key aspects of this new model do mimic human psoriasis. Mice show skin-predominant but systemic inflammation as well as the prominent neutrophilia and T cell-derived IL-17 and reversal of disease with anti-IL-17, all of which have been observed in human psoriasis (7). A hallmark of human psoriatic skin is a Th1- and Th17-biased cytokine milieu $(8,9)$. Recently the importance of IL17A as a key driver of psoriasis has become clear, based on IL-17directed therapies. In this study, we show that hCD1Tg HJ1Tg $A p o e^{-/-}$mice also have heightened Th17 response, which reflects the cytokine secretion pattern of HJ1 T cells. It is noteworthy that $\mathrm{HJ} 1 \mathrm{~T}$ cells represent only $1 \mathrm{CD} 1 \mathrm{~b}$-autoreactive TCR; thus, caution must be observed when generalizing these findings to other group 1 CD1-restricted T cells. However, based on the identification of human CD1b-autoreactive $\mathrm{T}$ cells that recognize the same lipid antigens defined here (19), it is plausible to suggest that a phospholipid-specific T cell response could exist in humans. Additionally, CD1b is expressed on a very specific subset of dermal DCs; other antigen-presenting cells (APCs) might respond differently to hyperlipidemia, which could elicit a more Th1-biased response from T cells. Furthermore, it is known that dermal DCs in the skin are capable of skewing T cell responses to both the Th17 and Th1 lineages as a result of IL-23 and IL-12 secretion (55). Nonetheless, since phospholipids infiltrate skin and IL-17A-producing group 1 CD1-autoreactive $\mathrm{T}$ cells were increased in hyperlipidemic mice that contained polyclonal CD1b- and CD1c-autoreactive $\mathrm{T}$ cells

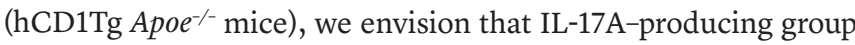
1 CD1-autoreactive $\mathrm{T}$ cells might play a role in hyperlipidemiainduced skin inflammation.

Our findings demonstrate that lack of ApoE leads to the accumulation of phospholipids and cholesterol species in the skin. This result is in agreement with both human hyperlipidemia syndromes and a previous study, which reported that Apoe $^{-/-}$mice develop cutaneous xanthomas (deposits of cho- lesterol-rich material in the skin) (56). Since ApoE is expressed at appreciable levels in the skin, it can be speculated that its absence can lead to the accumulation of lipids, which would otherwise be exported to the liver by ApoE through the process of reverse cholesterol transport. It has been demonstrated that hyperlipidemia can affect DC maturation while retaining DC antigen processing and presentation capacity to prime $\mathrm{T}$ cells (39-41). Indeed, the expression of CD86 is increased in dermal DCs of hCD1Tg HJ1Tg Apoe ${ }^{-/-}$mice. Additionally, our results show that hyperlipidemic serum causes DCs to produce more IL-6, which subsequently enhances IL-17A production by HJ1 T cells. ApoE has been shown to facilitate the uptake and delivery of exogenous lipid antigens for presentation by CD1b (57). However, since there is no difference in the ability of HJ1 T cells to produce IL-17A when cocultured with either hCD1Tg or hCD1Tg Apoe - $^{-}$DCs, we speculate that alternative antigen internalization pathways, such as scavenger receptor-mediated lipid uptake, compensate for the lack of ApoE. Indeed, hyperlipidemia has been demonstrated to cause upregulation of scavenger receptors on DCs.

We detected a higher frequency of CD1a- and CD1b-restricted $\mathrm{T}$ cells in the blood of psoriatic patients compared with normal individuals. Even though it is currently unknown whether psoriasis leads to hyperlipidemia or the other way around, our studies clearly indicate that both these conditions are interlinked and that hyperlipidemia could be a risk factor for the development of psoriasis. Our results are most consistent with a scenario that, under conditions of hyperlipidemia, lipids accumulate in the skin, activating CD1b-autoreactive $\mathrm{T}$ cells. Autoantigens such as phospholipids and cholesterol species are likely presented by CD1b to activate these $\mathrm{T}$ cells, resulting in the secretion of Th17 cytokines, which contribute to skin pathology. Since HJ1 $\mathrm{T}$ cells exhibit a preactivated phenotype in naive mice, they can be directly activated in the skin by CD1b-expressing DCs. Alternatively, they might be activated in the LNs by CD1b-expressing DCs carrying lipids from the skin, or both these mechanisms could be at play. Overall, this study not only sheds light on the role of group 1 CD1-autoreactive $\mathrm{T}$ cells in a chronic inflammatory disease, but also identifies a potential cause of the inflammatory process that could serve as a link among psoriasis, hyperlipidemia, and cardiovascular diseases.

\section{Methods}

Mice. Apoe $e^{-/}$mice were purchased from the Jackson Laboratory. hCD1Tg and hCD1Tg HJ1Tg mice have been described previously (37, 38) and were crossed with Apoe $e^{-/-}$mice to generate hCD1Tg Apoe $e^{-/-}$and hCD1Tg HJ1Tg Apoe $e^{--}$mice, respectively. All animals were housed in a specific pathogen-free facility at Northwestern University.

Cell preparations. Hepatic leukocyte and LN cell suspensions were prepared by manually grinding the organs in HBSS containing $2 \%$ FBS and 1\% HEPES. Liver suspensions were passed through a $37 \%$ Percoll gradient to separate leukocytes from hepatocytes. Red blood cells were lysed using Gey's balanced salt solution. Dermal cells were isolated from the skin by incubating skin sections in $2.5 \mathrm{U} / \mathrm{ml}$ dispase II (STEMCELL Technologies) in a $37^{\circ} \mathrm{C}$ shaking incubator for 3 hours and subsequently separated from the epidermis. Dermal layers were then cut into small pieces and digested further with $1 \mathrm{mg} / \mathrm{ml}$ collage- 
nase IV (Thermo Fisher) and $167 \mu \mathrm{g} / \mathrm{ml}$ DNase I (Thermo Fisher) in a $37^{\circ} \mathrm{C}$ shaking incubator for 30 minutes.

In vivo anti-IL-17A treatment. For in vivo anti-IL-17A blocking experiments, hCD1Tg HJ1Tg Apoe $e^{-/}$mice were injected (i.v.) with either $200 \mu \mathrm{g}$ of anti-IL-17A (BioXcell) or an isotype control (BioXcell) $\mathrm{Ab}$ twice per week, once i.v. and once i.p. This injection protocol was repeated for 3 weeks.

Flow cytometry. For cell-surface staining, cells were preincubated with purified 2.4G2 mAbs and then stained with the appropriate combinations of mAbs. For intracellular cytokine staining, cells were stimulated with PMA and ionomycin for 4 hours, followed by addition of brefeldin A for 2 hours. Cells were surface stained and fixed with $4 \%$ paraformaldehyde and permeabilized with $0.1 \%$ saponin, followed by anti-cytokine mAb staining. Intracellular FoxP3 staining was performed using the eBioscience FoxP3 Staining Buffer Set. Flow cytometry was performed with a FACSCanto II (BD Biosciences) and analyzed using FlowJo software (Tree Star). Anti-CD45 (30-F11), integrin $\alpha_{6}$ (GoH3), integrin $\beta_{1}$ (HM $\left.31-1\right)$, CD34 (HM34), Sca-1 (D7), TCR- $\beta$ (H57597), Vß2 (B20.6), CD69 (H1.2F3), CD44 (IM7), CD5 (53-7.3), CD11b (M1/70), CD11c (N418), CD207 (4C7), Ly6G (1A8), B220 (RA3-6B2), F4-80 (BM8), CD1b (SN-13), CD86 (GL-1), IFN- $\gamma$ (XMG1.2), IL-17A (TC11-18H10.1), CD4 (GK1.5), CD8 (53-6.7), and FoxP3 (MF-14) Abs were purchased from BioLegend. CD1b-loaded phospholipid tetramers were made in the lab as previously described (19) from biotinylated CD1b protein provided by the NIH Tetramer Core Facility.

DC and T cell coculture assays. BMDCs were pretreated with Pam${ }_{3}$ Cys $(100 \mathrm{ng} / \mathrm{ml})$ overnight and washed thoroughly before coculturing with enriched $\mathrm{T}$ cells from indicated mouse strains. Hepatic $\mathrm{T}$ cells were enriched using Miltenyi MACS magnetic separation. Briefly, cells were incubated with biotinylated Abs against CD11b, MHC II, Ter119, and B220, followed by incubation with streptavidin-coupled magnetic beads according to the manufacturer's protocol. $1 \times 10^{5} \mathrm{BMDCs}$ and 2 to $4 \times 10^{5} \mathrm{~T}$ cells were added to each well and cultured for 48 hours. IFN- $\gamma$ and IL-17A in culture supernatants were measured by ELISA. mAb pairs for ELISA were purchased from BD Biosciences - Pharmingen. For ELISPOT assays, BMDC and T cell cocultures were incubated for 18 hours in anti-IL-17A-coated plates. The same capture and secondary anti-IL-17A mAbs were used for both ELISA and ELISPOT assays. Cytokine-producing cells were quantified using an ImmunoSpot reader (Cellular Technology Ltd.). For the assay with hyperlipidemic serum, CD1b- and CD1c-expressing DCs were isolated from the LNs of indicated mice after inoculating them with granulocyte-macrophageCSF-transfected (GM-CSF-transfected) B16 cells for 8 to 10 days to increase the total number of DCs. These DCs were cocultured with $4 \times$ $10^{5}$ enriched hepatic HJ1 T cells from hCD1Tg HJ1Tg Rag/- mice for 48 hours in the presence of either normal or hyperlipidemic mouse serum. ELISA for IFN- $\gamma$ and IL-17A was subsequently performed.

$m R N A$ detection. Tissues were isolated and snap-frozen in liquid nitrogen and stored at $-80^{\circ} \mathrm{C}$ until use. Subsequently, tissues were thawed, homogenized, and centrifuged at 10,000 $\mathrm{g}$ for $5 \mathrm{~min}$ utes. Lysates were collected and mRNA extracted using the QIAGEN RNeasy Mini Kit. Subsequently, RNA was reverse transcribed into cDNA using Superscript III. Primers (primer sequences have been listed in Supplemental Table 1) against targets of interest were mixed together with cDNA and iQ SYBR Green Supermix. Then quantitative PCR was performed using a Bio-Rad iQ5 cycler for detection of mRNA in tissue samples.
Histological staining. For aortic root sections, mice were perfused through the heart with PBS and then $4 \%$ paraformaldehyde plus 5\% sucrose solution for 10 minutes with each reagent. Subsequently, the heart was excised with intact innominate, left, and right subclavian arteries. Hearts were fixed further in $4 \%$ paraformaldehyde overnight and frozen in OCT. Aortic root sections were obtained using a cryostat. Each section was $10-\mu \mathrm{m}$ thick. The sections were then stained with oil red $\mathrm{O}$ solution. The plaque area was quantified from 3 aortic root sections as described previously (51) using Nikon-Elements software. For colon sections, the organ was isolated as previously described (58) and fixed in 10\% formalin and laid out in Swiss rolls for paraffin embedding. Paraffin sections were cut and stained with H\&E for examination of the tissue architecture. For histological analysis of mouse skin, samples were fixed in $10 \%$ neutral buffered formalin and embedded in paraffin. Sections of $5 \mu \mathrm{m}$ were acquired and deparaffinized. Sections were either stained with $\mathrm{H} \& \mathrm{E}$ or were blocked with $5 \%$ normal donkey serum and incubated with primary Abs (CD3, Gr-1, Ki67; Abcam). Subsequently, sections were washed and stained with secondary Abs conjugated to a fluorophore for immunofluorescence or secondary Ab staining was followed by incubation with Vectastain (Vector Laboratories). Finally, sections were incubated with biotin tyramide working solution and streptavidin-HRP. For IHC staining of human skin samples, sections were deparaffinized, followed by antigen retrieval in citrate buffer. Subsequently, sections were blocked and stained with primary Abs (CD1a, CD1b, or CD1c), then with secondary Abs (Dako Envision Labeled Polymer), and staining signal was detected using Dako DAB substrate solution. The slides were scanned using a TissueFAXS imaging system equipped with a Zeiss Axio microscope (TissueGnostics GmbH). The number of CD1a-, CD1b-, and CD1c-positive cells in the tissue sections was analyzed using HistoQuest Image Analysis software (TissueGnostics GmbH).

Lipid preparation and analysis. Lipids were either purchased from commercial sources or extracted in the lab. Cholesterol and bovine liver polar lipid extract were purchased from Avanti Polar Lipids. Fatty acid mixtures (FA1 containing saturated and monounsaturated fatty acids and FA2 containing polyunsaturated fatty acids) were purchased from Cayman Chemicals. Alternatively, lipids were extracted from mouse skin and liver tissues by homogenizing the samples in water, followed by addition of 1:2 chloroform/methanol. After shaking, 1:1 chloroform/water was added and contents were centrifuged at $200 \mathrm{~g}$ for 10 minutes. Subsequently, bottom layers were extracted with chloroform twice. Finally, the lipid-containing fractions were washed with a small volume of $1 \mathrm{M} \mathrm{KCl}$ and then water. The lipid phase was dried under a nitrogen stream, and the weight of dried lipid was determined. The composition of lipids was determined at the Kansas Lipidomics Research Center (Kansas State University, Manhattan, Kansas, USA) either by tandem mass spectrometry or gas chromatography.

Plate-bound activation assay. The HJ1 and LN1-7 T cell hybridoma lines were generated as previously described (59). From 1.5 to $2 \mu \mathrm{g} /$ $\mathrm{ml}$ of soluble CD1b protein was incubated (in PBS) with $10 \mu \mathrm{g} / \mathrm{ml} \mathrm{lipid}$ antigens for 4 hours. Then, 96-well Immulon Plates (Thermo Fisher Scientific) were coated for 2 hours at $37^{\circ} \mathrm{C}$ with $100 \mu$ of CD1b protein loaded with or without lipid. Subsequently, wells were washed 3 times with RPMI-10 media, and $5 \times 10^{4} \mathrm{HJ} 1$ or LN1-7 T cell hybridomas were added per well. Twenty-four hours later, IL-2 in the culture supernatant was measured by ELISA. IL-2 mAb pair for ELISA was purchased from BD Biosciences - Pharmingen. 
Human sample acquisition and processing. The Skin Disease Research Center (SDRC) at Northwestern University provided deidentified skin samples from psoriatic patients who were hyperlipidemic and normal individuals for histological staining. PBMCs from psoriatic patients were either acquired through the SDRC or from J.E. Gudjonsson. PMBCs were isolated within 24 hours of blood draw. Samples from both male and female subjects were used. Psoriatic patients were off both topical and systemic treatments at the time of blood draw and had moderate to severe disease. From 30 to $50 \mathrm{ml}$ of whole blood was obtained by venipuncture using heparinized blood collection tubes (BD Biosciences), and PBMCs were isolated using Histopaque-1077 (Sigma-Aldrich). Approximately 1 to $2 \times 10^{6} \mathrm{PBMCs} / \mathrm{ml}$ of blood were isolated, as determined by counting using a hemocytometer. $\mathrm{T}$ cells from PBMCs were enriched using biotinylated anti-CD3 Abs (eBioscience) and subsequently by positive selection with Streptavidin MicroBeads following the manufacturer's protocol (Miltenyi Biotec). T cells were then immediately frozen. Autologous monocyte-derived DCs (moDCs) were allowed to adhere to the surfaces of $25-\mathrm{cm}^{2}$ flasks (Falcon) for 90 minutes in RPMI-5 media (RPMI 1640 supplemented with 5\% human serum, $1 \%$ penicillin-streptomycin, $20 \mathrm{mM}$ L-glutamine, and $50 \mu \mathrm{M}$ 2-mercaptoethanol) and $20 \mathrm{ng} / \mathrm{ml}$ human GM-CSF (Shenandoah Biotechnology). Subsequently, nonadherent cells were removed and adherent cells were cultured in RPMI-5 with $20 \mathrm{ng} / \mathrm{ml}$ of human IL-4 (Shenandoah Biotechnology) and GM-CSF for 3 days. Autologous mo-DCs $\left(2 \times 10^{5}\right.$ cells $)$ were then cultured with thawed T cells $\left(2 \times 10^{6}\right.$ cells) in 24-well tissue culture plates. After 7 days, cells were stimulated again with mo-DCs. At day 14, T cells were used in ELISPOT assays.

Human T cell ELISPOT assays. Human T cells cultured for 14 days with autologous mo-DCs were cocultured with K562 and K562-CD1 transfectants (K562-CD1a, K562-CD1b, and K562-CD1c) for 18 hours (16). About $0.5 \times 10^{5}$ to $1 \times 10^{5} \mathrm{~T}$ cells were cultured with $2.5 \times 10^{4}$ to 5 $\times 10^{4} \mathrm{~K} 562$ cells in ELISPOT plates precoated with anti-IL-2 Abs (Millipore). Coating and detection Abs specific for human IL-2 were purchased from Mabtech. Cytokine-producing cells were quantified using an ImmunoSpot reader.

Human $T$ cell clones and tetramer staining. The polyclonal human $\mathrm{T}$ cell lines A25 and BC8 were originally derived from random blood bank donors by selection for recognition of CD1b loaded with phospholipids (19). For flow cytometry, CD1b tetramers were mock treated or loaded with synthetic C16 C18:1 PE (Avanti 850757c) or synthetic C18 C18:1 PG (Avanti 840503). A25 or BC8 cells were incubated with tetramers for 30 minutes at room temperature, washed, and analyzed on an LSR Fortessa Flow Cytometer (BD).

Statistics. Statistical analysis was performed with unpaired Student's $t$ test for 2-group comparisons or 1-way ANOVA for more than 2 group comparisons, followed by Bonferroni's post-hoc test. Group 1 CD1-restricted autoreactive $\mathrm{T}$ cell responses in cohorts of healthy individuals and patients with psoriasis were analyzed using the MannWhitney $U$ test. All statistical analyses were performed with Prism software. $P<0.05$ was considered significant. Statistically significant differences are noted.

Study approval. All animal studies were reviewed and approved by the Institutional Animal Care and Use Committee at Northwestern University. Normal and psoriatic blood samples were acquired from volunteers, and informed consent was obtained from participating donors prior to their inclusion in this study. The institutional review boards at Northwestern University and the University of Michigan Medical School approved all human studies, which were conducted according to the Declaration of Helsinki Principles.

\section{Author contributions}

SB and CRW designed experiments, analyzed the data, and wrote the manuscript. SB, YH, HZ, and LC performed experiments. IVR, DBM, and JEG participated in the preparation of the manuscript. IVR made CD1b-phospholipid tetramers. JEG provided blood samples from psoriasis patients.

\section{Acknowledgments}

We thank Jenny Gumperz for providing K562 transfectants, Catherine Reardon and Nicole Ward for protocols and advice, the Northwestern University Mouse Histology and Phenotyping Laboratory for histology services, Northwestern SDRC for providing formalin-fixed skin biopsies from normal and psoriatic individuals and whole blood from psoriatic patients, the Northwestern Cell Imaging Facility for assisting with skin imaging and quantifying group 1 CD1-expressing cells, the Kansas Lipidomics Research Center (Kansas State University) for mass spectrometric and gas chromatographic analyses for determining lipid composition in tissues, and the NIH Tetramer Core Facility for CD1b protein. This work was supported by NIH R01 AI05746, R21 HL112186, R21 AI117238, and a pilot and feasibility grant from SDRC at Northwestern University (5P30AR057216-08 to CRW). JEG is supported by the Kenneth and Frances Eisenberg Emerging Scholar Award of the A. Alfred Taubman Medical Research Institute, NIH K08 AR060802, and NIH RO1 AR069071.

Address correspondence to: Chyung-Ru Wang, Department of Microbiology and Immunology, Northwestern University, 320 E. Superior Street, Searle 3-401, Chicago, Illinois 60611, USA. Phone: 312.503.9748; E-mail: chyung-ru-wang@northwestern.edu.

HZ's present address is: Department of Surgery, Thomas E. Starzl Transplantation Institute, University of Pittsburgh Medical Center, Pittsburgh, Pennsylvania, USA.
1. Ogden CL, Carroll MD, Fryar CD, Flegal KM. Prevalence of obesity among adults youth: United States, 2011-2014. NCHS Data Brief. 2015;(219):1-8.

2. Nelson RH. Hyperlipidemia as a risk factor for cardiovascular disease. Prim Care. 2013;40(1):195-211.

3. Azfar RS, Gelfand JM. Psoriasis and metabolic disease: epidemiology and pathophysiology. Curr Opin Rheumatol. 2008;20(4):416-422.

4. Pietrzak A, Michalak-Stoma A, Chodorowska G,
Szepietowski JC. Lipid disturbances in psoriasis: an update. Mediators Inflamm. 2010;2010.

5. Carrascosa JM, et al. Obesity and psoriasis: inflammatory nature of obesity, relationship between psoriasis and obesity, and therapeutic implications. Actas Dermosifiliogr. 2014;105(1):31-44.

6. Armstrong AW, Harskamp CT, Armstrong EJ. The association between psoriasis and obesity: a systematic review and meta-analysis of observational studies. Nutr Diabetes. 2012;2:e54.
7. Nestle FO, Kaplan DH, Barker J. Psoriasis. NEng JMed. 2009;361(5):496-509.

8. Cai Y, Fleming C, Yan J. New insights of T cells in the pathogenesis of psoriasis. Cell Mol Immunol. 2012;9(4):302-309.

9. Coimbra S, Figueiredo A, Castro E, RochaPereira P, Santos-Silva A. The roles of cells and cytokines in the pathogenesis of psoriasis. Int $J$ Dermatol. 2012;51(4):389-395; quiz 395.

10. Gaspari AA, Tyring S. New and emerging biologic 
therapies for moderate-to-severe plaque psoriasis: mechanistic rationales and recent clinical data for IL-17 and IL-23 inhibitors. Dermatol Ther. 2015;28(4):179-193.

11. Garnock-Jones KP. Secukinumab: a review in moderate to severe plaque psoriasis. Am JClin Dermatol. 2015;16(4):323-330.

12. Morizane S, Gallo RL. Antimicrobial peptides in the pathogenesis of psoriasis. J Dermatol. 2012;39(3):225-230.

13. Lande R, et al. The antimicrobial peptide LL37 is a T-cell autoantigen in psoriasis. Nat Commun. 2014;5:5621.

14. Arakawa A, et al. Melanocyte antigen triggers autoimmunity in human psoriasis. J Exp Med. 2015;212(13):2203-2212.

15. Nishimoto $S$, et al. Th17 cells carrying TCR recognizing epidermal autoantigen induce psoriasis-like skin inflammation. JImmunol. 2013;191(6):3065-3072.

16. de Jong A, Peña-Cruz V, Cheng TY, Clark RA, Van Rhijn I, Moody DB. CD1a-autoreactive T cells are a normal component of the human $\alpha \beta$ T cell repertoire. Nat Immunol.2010;11(12):1102-1109.

17. de Jong A, et al. CD1a-autoreactive T cells recognize natural skin oils that function as headless antigens. Nat Immunol. 2014;15(2):177-185.

18. de Lalla C, et al. High-frequency and adaptivelike dynamics of human $\mathrm{CD} 1$ self-reactive $\mathrm{T}$ cells. Eur J Immunol. 2011;41(3):602-610.

19. Van Rhijn I, et al. Human autoreactive T cells recognize CD1b and phospholipids. Proc Natl Acad Sci USA. 2016;113(2):380-385.

20. Calabi F, Bradbury A. The CD1 system. Tissue Antigens. 1991;37(1):1-9.

21. Van Kaer L, Parekh VV, Wu L. Invariant natural killer $\mathrm{T}$ cells as sensors and managers of inflammation. Trends Immunol. 2013;34(2):50-58.

22. Van Rhijn I, Godfrey DI, Rossjohn J, Moody DB. Lipid and small-molecule display by CD1 and MR1. Nat Rev Immunol. 2015;15(10):643-654.

23. Siddiqui S, Visvabharathy L, Wang CR. Role of Group 1 CD1-Restricted T Cells in Infectious Disease. Front Immunol. 2015;6:337.

24. Dougan SK, Kaser A, Blumberg RS. CD1 expression on antigen-presenting cells. Curr Top Microbiol Immunol. 2007;314:113-141.

25. Vincent MS, Xiong X, Grant EP, Peng W, Brenner MB. CD1a-, b-, and c-restricted TCRs recognize both self and foreign antigens. J Immunol. 2005;175(10):6344-6351.

26. Shamshiev A, Donda A, Carena I, Mori L, Kappos L, De Libero G. Self glycolipids as T-cell autoantigens. Eur J Immunol. 1999;29(5):1667-1675.

27. Shamshiev A, et al. The alphabeta $\mathrm{T}$ cell response to self-glycolipids shows a novel mechanism of CD1b loading and a requirement for complex oligosaccharides. Immunity. 2000;13(2):255-264.

28. Shamshiev A, Gober HJ, Donda A, Mazorra Z, Mori L, De Libero G. Presentation of the same glycolipid by different CD1 molecules. J Exp Med . 2002;195(8):1013-1021.

29. Macho-Fernandez E, Brigl M. The extended family of CD1d-restricted NKT cells: sifting through a mixed bag of TCRs, antigens, and functions. Front Immunol. 2015;6:362.

30. Cheung KL, et al. Psoriatic T cells recognize neolipid antigens generated by mast cell phospholipase delivered by exosomes and presented by CD1a. J Exp Med. 2016;213(11):2399-2412.

31. Mansour S, et al. Cholesteryl esters stabilize human CD1c conformations for recognition by self-reactive T cells. Proc Natl Acad Sci U S A. 2016;113(9):E1266-E1275.

32. Peternel S, Kastelan M. Immunopathogenesis of psoriasis: focus on natural killer T cells. JEur Acad Dermatol Venereol. 2009;23(10):1123-1127.

33. Nickoloff BJ, Bonish B, Huang BB, Porcelli SA. Characterization of a $\mathrm{T}$ cell line bearing natural killer receptors and capable of creating psoriasis in a SCID mouse model system. J Dermatol Sci. 2000;24(3):212-225.

34. Gilhar A, et al. Psoriasis is mediated by a cutaneous defect triggered by activated immunocytes: induction of psoriasis by cells with natural killer receptors. J Invest Dermatol. 2002;119(2):384-391.

35. Li B, et al. Transcriptome analysis of psoriasis in a large case-control sample: RNA-seq provides insights into disease mechanisms. J Invest Dermatol. 2014;134(7):1828-1838.

36. Kim JH, et al. CD1a on Langerhans cells controls inflammatory skin disease. Nat Immunol. 2016;17(10):1159-1166.

37. Felio K, et al. CD1-restricted adaptive immune responses to Mycobacteria in human group $1 \mathrm{CD} 1$ transgenic mice. J Exp Med 2009;206(11):2497-2509.

38. Li S, Choi HJ, Felio K, Wang CR. Autoreactive CD1b-restricted T cells: a new innate-like T-cell population that contributes to immunity against infection. Blood. 2011;118(14):3870-3878.

39. Zaguri R, et al. 'Danger' effect of low-density lipoprotein (LDL) and oxidized LDL on human immature dendritic cells. Clin Exp Immunol. 2007;149(3):543-552.

40. Alderman CJ, Bunyard PR, Chain BM, Foreman JC, Leake DS, Katz DR. Effects of oxidised low density lipoprotein on dendritic cells: a possible immunoregulatory component of the atherogenic microenvironment? Cardiovasc Res. 2002;55(4):806-819.

41. Nickel T, et al. oxLDL uptake by dendritic cells induces upregulation of scavenger-receptors, maturation and differentiation. Atherosclerosis. 2009;205(2):442-450.

42. Porcelli S, Brenner MB, Greenstein JL, Balk SP, Terhorst C, Bleicher PA. Recognition of cluster of differentiation 1 antigens by human CD4-CD8-cytolytic T lymphocytes. Nature. 1989;341(6241):447-450.
43. Roura-Mir C, et al. Mycobacterium tuberculosis regulates $\mathrm{CD} 1$ antigen presentation pathways through TLR-2. JImmunol. 2005;175(3):1758-1766.

44. Yakimchuk K, et al. Borrelia burgdorferi infection regulates $\mathrm{CD} 1$ expression in human cells and tissues via IL1- $\beta$. Eur J Immunol. 2011;41(3):694-705.

45. Lepore $\mathrm{M}$, et al. A novel self-lipid antigen targets human $\mathrm{T}$ cells against $\mathrm{CD} 1 \mathrm{c}(+)$ leukemias. JExp Med. 2014;211(7):1363-1377.

46. Bagchi S, Li S, Wang CR. CD1b-autoreactive T cells recognize phospholipid antigens and contribute to antitumor immunity against a CD1b(+) T cell lymphoma. Oncoimmunology. 2016;5(9):e1213932.

47. Gudjonsson JE, Johnston A, Dyson M, Valdimarsson H, Elder JT. Mouse models of psoriasis. J Invest Dermatol. 2007;127(6):1292-1308.

48. Flutter B, Nestle FO. TLRs to cytokines: mechanistic insights from the imiquimod mouse model of psoriasis. Eur JImmunol. 2013;43(12):3138-3146.

49. Bergstresser PR, Cruz PD, Takashima A. Dendritic epidermal $\mathrm{T}$ cells: lessons from mice for humans. J Invest Dermatol. 1993;100(1):80S-83S.

50. Tupin E, et al. CD1d-dependent activation of NKT cells aggravates atherosclerosis. J Exp Med. 2004;199(3):417-422.

51. VanderLaan PA, et al. Characterization of the natural killer T-cell response in an adoptive transfer model of atherosclerosis. Am J Pathol. 2007;170(3):1100-1107.

52. Major AS, et al. Quantitative and qualitative differences in proatherogenic NKT cells in apolipoprotein E-deficient mice. Arterioscler Thromb Vasc Biol. 2004;24(12):2351-2357.

53. Aslanian AM, Chapman HA, Charo IF. Transient role for CD1d-restricted natural killer T cells in the formation of atherosclerotic lesions. Arterioscler Thromb Vasc Biol. 2005;25(3):628-632.

54. Taleb S, Tedgui A, Mallat Z. IL-17 and Th17 cells in atherosclerosis: subtle and contextual roles. Arterioscler Thromb Vasc Biol. 2015;35(2):258-264.

55. Chu CC, Di Meglio P, Nestle FO. Harnessing dendritic cells in inflammatory skin diseases. Semin Immunol. 2011;23(1):28-41.

56. Moghadasian MH, Nguyen LB, Shefer S, McManus BM, Frohlich JJ. Histologic, hematologic, and biochemical characteristics of apo E-deficient mice: effects of dietary cholesterol and phytosterols. Lab Invest. 1999;79(3):355-364.

57. van den Elzen P, et al. Apolipoprotein-mediated pathways of lipid antigen presentation. Nature. 2005;437(7060):906-910.

58. Liao CM, Zimmer MI, Shanmuganad S, Yu HT, Cardell SL, Wang CR. dysregulation of CD1drestricted type ii natural killer $\mathrm{T}$ cells leads to spontaneous development of colitis in mice. Gastroenterology. 2012;142(2):326-34.e1.

59. Canaday DH. Production of CD4 ${ }^{+}$and $\mathrm{CD} 8^{+} \mathrm{T}$ cell hybridomas. Methods Mol Biol. 2013;960:297-307. 